\title{
Plume et mode à la Belle Époque
}

Les plumassiers parisiens face à la question animale

\section{Anne Monjaret}

\section{OpenEdition}

Journals

Édition électronique

URL : https://journals.openedition.org/tc/3952

DOI : $10.4000 /$ tc.3952

ISSN : 1952-420X

Éditeur

Éditions de l'EHESS

\section{Édition imprimée}

Date de publication : 31 décembre 2008

Pagination : 228-255

ISSN : 0248-6016

Référence électronique

Anne Monjaret, «Plume et mode à la Belle Époque », Techniques \& Culture [En ligne], 50 | 2008, mis en ligne le 31 décembre 2010, consulté le 29 septembre 2022. URL : http://journals.openedition.org/tc/ 3952 ; DOI : https://doi.org/10.4000/tc.3952 


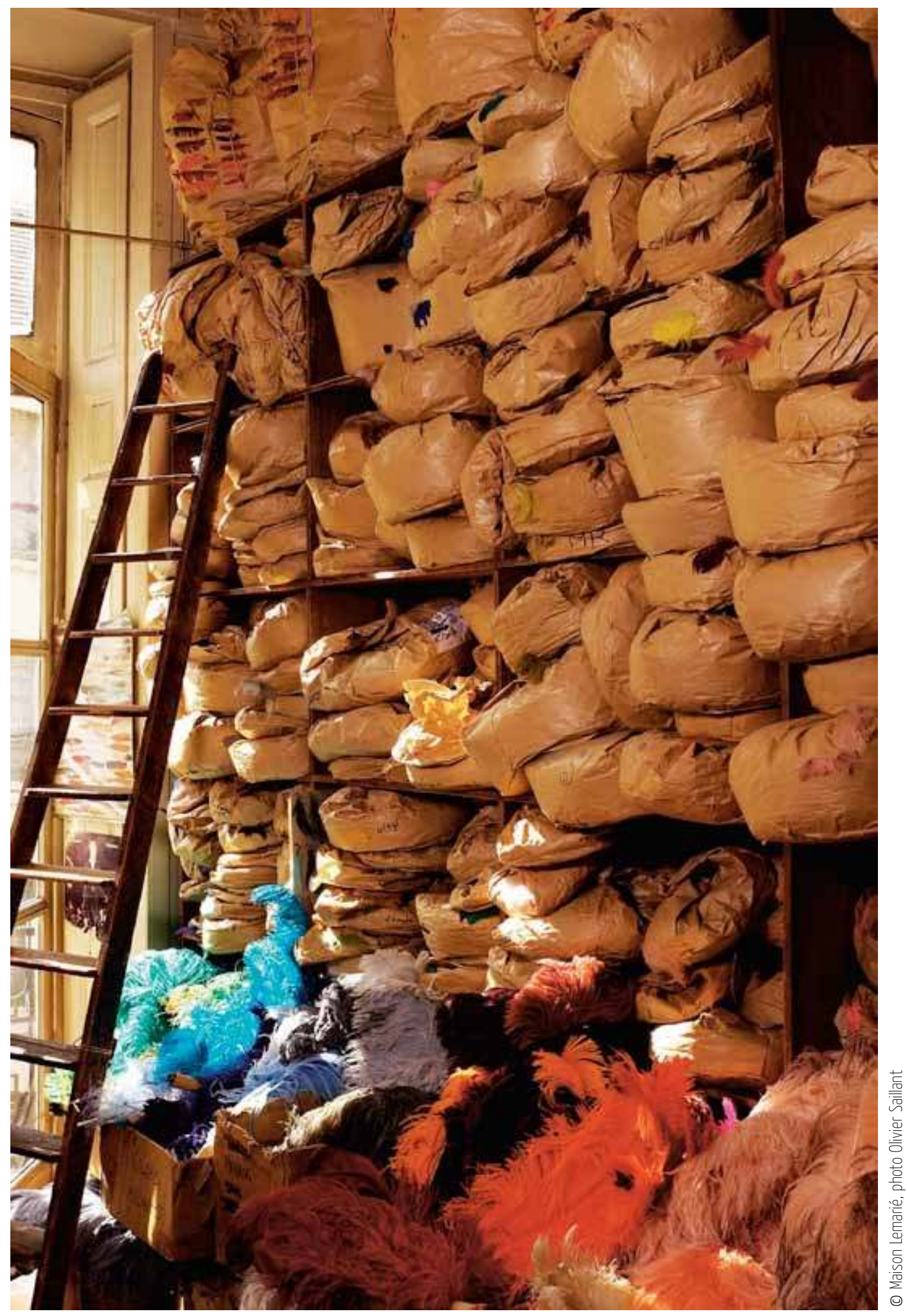


CERLIS, CNRS, Université Paris Descartes anne.monjaret@parisdescartes.fr

\title{
PLUME ET MODE À LA BELLE ÉPOQUE
}

\section{Les plumassiers parisiens face à la question animale}

\author{
Au tournant du $\mathrm{XIX}^{\mathrm{e}}$ et du $\mathrm{XX}^{\mathrm{e}}$ siècle, les oiseaux, sauvages et exotiques, ont été \\ exploités massivement par la mode parisienne pour garnir les chapeaux des femmes. \\ Aujourd'hui, respect de la nature et protection animale modèlent les pratiques \\ contemporaines des rares plumassiers parisiens qui travaillent encore.
}

La plume, matière première d'origine animale, a toujours été utilisée, en France et ailleurs, par les professionnels de la mode et du spectacle pour garnir et embellir les vêtements et leurs accessoires, en particulier féminins. C'est le chapeau arboré par les femmes dans les lieux publics qui nous paraît le mieux incarner l'histoire de cette production plumassière artisanale (Bolomier 1993; Monjaret 1995, 1998). Les plumassiers, fournisseurs ou fabricants, industriels ou artisans ${ }^{1}$ travaillant pour et dans la mode, doivent répondre à la demande d'une clientèle exigeante et s'adapter aux tendances imposées par la haute couture tout en sachant proposer leurs nouveautés. Cette adaptabilité n'est pas propre à ce corps de métier. Les plumassiers « font » et « défont » la mode au même titre que tous les artisans de l'élégance, qui, comme eux, jouent avec les formes, les couleurs et les matières pour renouveler chaque saison leur collection et profiter des engouements.

Ce qui les distingue est d'un autre ordre: ils travaillent la plume, transforment la nature en parure. L'usage de plumages et d'oiseaux naturalisés ou recomposés à des fins d'ornement intrigue et surtout interroge sur les 


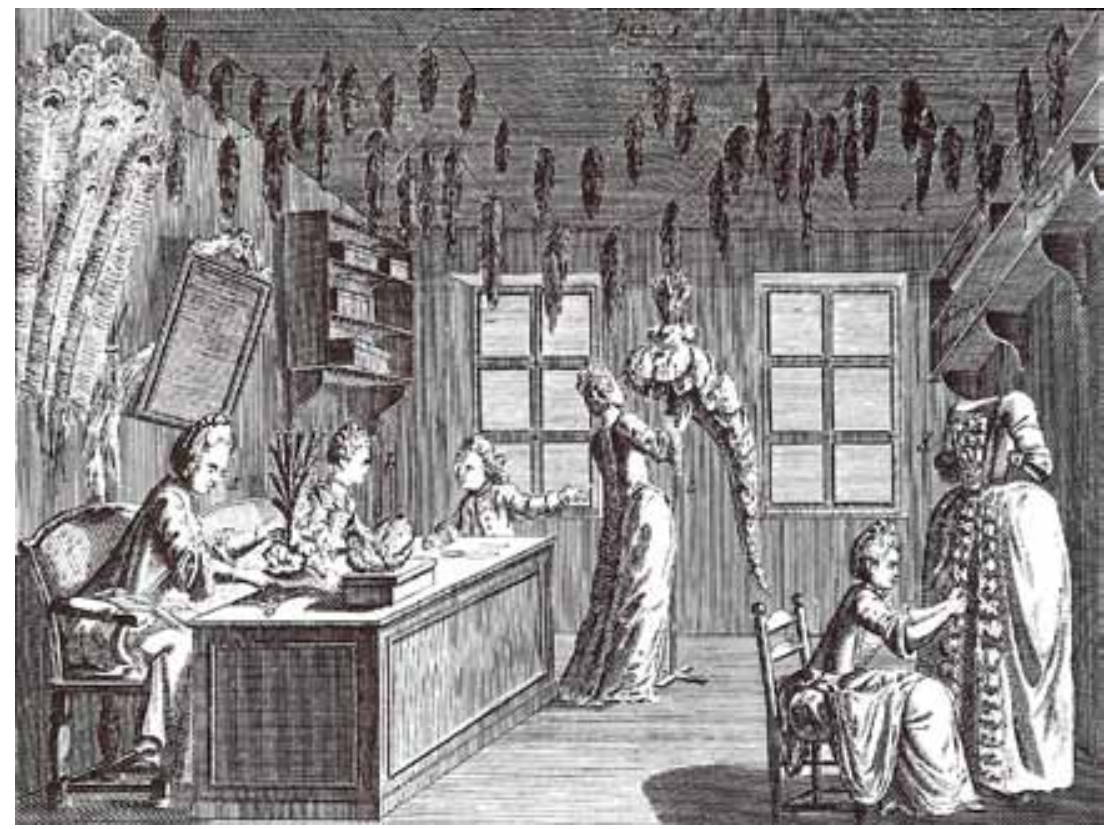

\section{Des espaces de travail}

Les locaux des plumassiers connaissaient et connaissent encore une distribution des pièces en accord avec l'activité qui y est exercée: vente, administration, production (ateliers et réserves). Chez ces professionnels, la plume est maîtresse, elle est partout, stockée dans des tiroirs ou des sacs papiers, accrochée au plafond ou au mur. Il se dégage fréquemment de ces ateliers des odeurs de cire, de camphre et d'antimite qui, mêlées au foisonnement de couleurs et de formes, donne au visiteur l'impression de pénétrer dans un univers « exotique » qui a peu bougé depuis un siècle. L'intérieur du plumassier-panachier présenté dans l'Encyclopédie de Diderot et d'Alembert et daté du XVIII e siècle apparait quant à lui presque sobre comparé à ceuX des XIXe et XXe siècles. Pourtant, leur parenté est bien réelle, il suffit de s'intéresser à la variété et à la sophistication de leur production pour s'en persuader.

(Fig. 1) espèces utilisées, exotiques ou domestiques, et par là sur la provenance de ces dernières. Il nous apparaît alors nécessaire de nous arrêter sur la particularité de la matière utilisée et travaillée. En effet, quelques questions s'imposent quant à l'utilisation de cette matière animale: comment s'est joué au cours des XIX et XX ${ }^{e}$ siècles le rapport entre industrie plumassière, ornithologie et protection animale? Quels effets cela a-t-il pu avoir sur ces professionnels français, leurs discours à propos de leur pratique, l'exercice et le contenu de leur métier? Autant de points à explorer qui nourrissent une réflexion sur les rapports entre l'homme et l'animal (Pujol $\&$ Carbone 1990), sur les conséquences économiques et sociales de la maîtrise humaine de la nature, et sur ce que nous pouvons nommer l'éveil d'une sensibilité pour le sort réservé aux animaux.

Les savoirs techniques des plumassiers artisans d'hier et d'aujourd'hui semblent immuables, et ce malgré des contextes sociaux et économiques distincts. Leurs productions artisanales, actes de création, traversent le temps sans profonds changements, en dehors de ceux qu'impose la mode (Fig. 1). Sur plus d'un siècle, le métier a finalement peu évolué. Ethnologue, c'est le constat de cette pérennité qui nous a incitée à un retour en arrière afin de mieux comprendre la situation présente de ces artisans de l'élégance $^{2}$. La démarche ethnologique que nous revendiquons ici ancre le présent dans le passé. L'étude de ce métier nécessite donc un va-et-vient historique et sociologique. Ainsi, si nous nous focalisons sur une période historique précise, c'est qu'elle nous apparaît comme une étape clé dans le processus de transformation qu'a connu le métier de plumassier et de justification de son exercice. Mais contrairement à l'ethno-histoire, nous ne cherchons pas à reconstituer pas à pas le cheminement d'un devenir, à faire une « sorte d'histoire à rebours » (Izard \& Wachtel 1991 : 338), mais plutôt à faire résonner un passé innovant avec un présent traditionnel, de 
vieux écrits avec une mémoire vivante. Et contrairement à l'anthropologie historique, nous ne nous arrêterons pas véritablement sur les «mentalités » de la Belle Époque, ni sur les évolutions lentes du métier (Schmitt 1992 : 339). Nous choisissons d'être sur les points de rupture et de tension.

Notre analyse se base non seulement sur les propos recueillis lors de nos enquêtes auprès de plumassiers parisiens, mais également sur des écrits datés du XIX et XXe siècles où partisans et détracteurs des plumassiers s'affrontent: d'un côté, des ornithologues, souvent membres du comité de défense des industries plumassières créé en 1908, et de l'autre des journalistes ou écrivains qui s'intéressent au milieu de la mode ou aux femmes.

\section{La mode en accusation}

Comme nous le relate un de ces auteurs du XIXe siècle, "les plumes d'oiseaux recueillies dans les pays américains, asiatiques, océaniens et africains, sont soumises aux aléas des préférences féminines, qui adopteront pendant une saison les oiseaux-mouches, et les lophophores, ou fixeront leurs choix sur la crosse et sur le paradis » (Calmettes s.d. : 224). Ce sont les plumes exotiques, rares et précieuses, qui sont au goût du jour et parent les chapeaux des dames du monde et d'autres classes sociales encore, soucieuses d'originalité (Fig. 2 et 3).

La plume est un ornement naturel de choix qui a, depuis toujours, suscité l'intérêt des paruriers (Lefèvre 1914), de leurs clients et clientes. Même si les motifs en plume se font plus ou moins discrets selon les périodes, cette mode féminine des chapeaux garnis et des toilettes de soirée connaît son âge d'or jusque dans les Années folles. Elle contribue au développement économique du secteur, chaque saison annonçant l'adoption d'une nouvelle espèce d'oiseaux, mais aussi au développement des remarques voire des critiques. C'est à ces réactions que nous nous intéresserons ici. Pourquoi cet usage des plumes est-il contesté? Quels sont les arguments déployés pour le condamner? Et par qui? Pour autant, notre réflexion s'inscrit davantage dans une ethnologie des techniques et des métiers que dans une sociologie des controverses qui décortiquerait précisément les arguments des débats. Ce qui nous occupe dans le cadre de cet article ${ }^{3}$, c'est de cerner le contexte qui a entraîné des changements techniques dans la production de ces artisans, de mesurer les conséquences locales d'un contexte politique, économique international sur la pratique de ces professionnels.

\section{Des chapeaux incriminés...}

En 1914, Edmond Lefèvre, ingénieur, président du comité d'ornithologie économique, comité de défense de l'industrie plumassière, conforte les propos de Pierre Calmettes, en citant ceux de Madame Celnart tirés de son Manuel des dames ou l'art de la toilette daté de 1827 qui notait: " Par une bizarrerie particulière, la mode a voulu, il y a peu de temps, qu'un

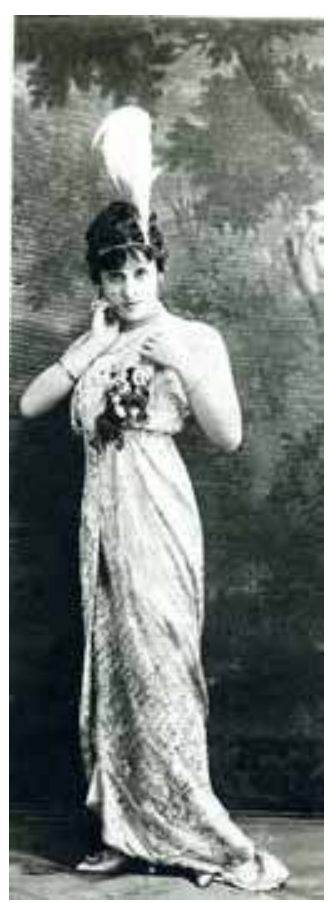

Les parures de plume dans la presse

À la Belle Époque, la presse qui poursuit son essor consacre des pages à la mode féminine, c'est le cas de la « revue hebdomadaire des actualités », Le Miroir, datée du dimanche 26 juillet 1914 dont sont extraites ces deux images. Les plumes de parure, de l'aigrette à l'éventail, ne sont pas absentes de ces pages.

(Fig. 2 et 3)

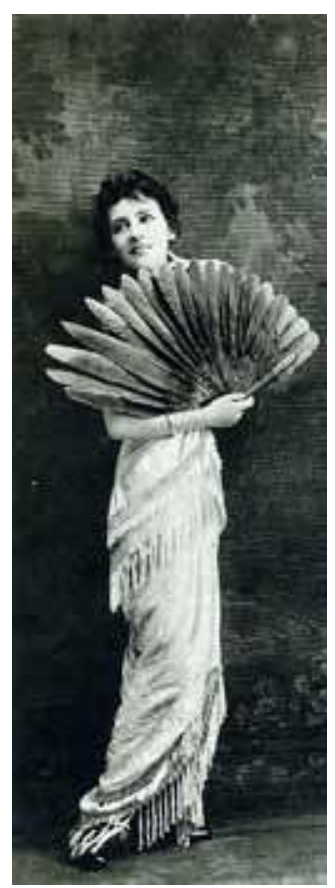



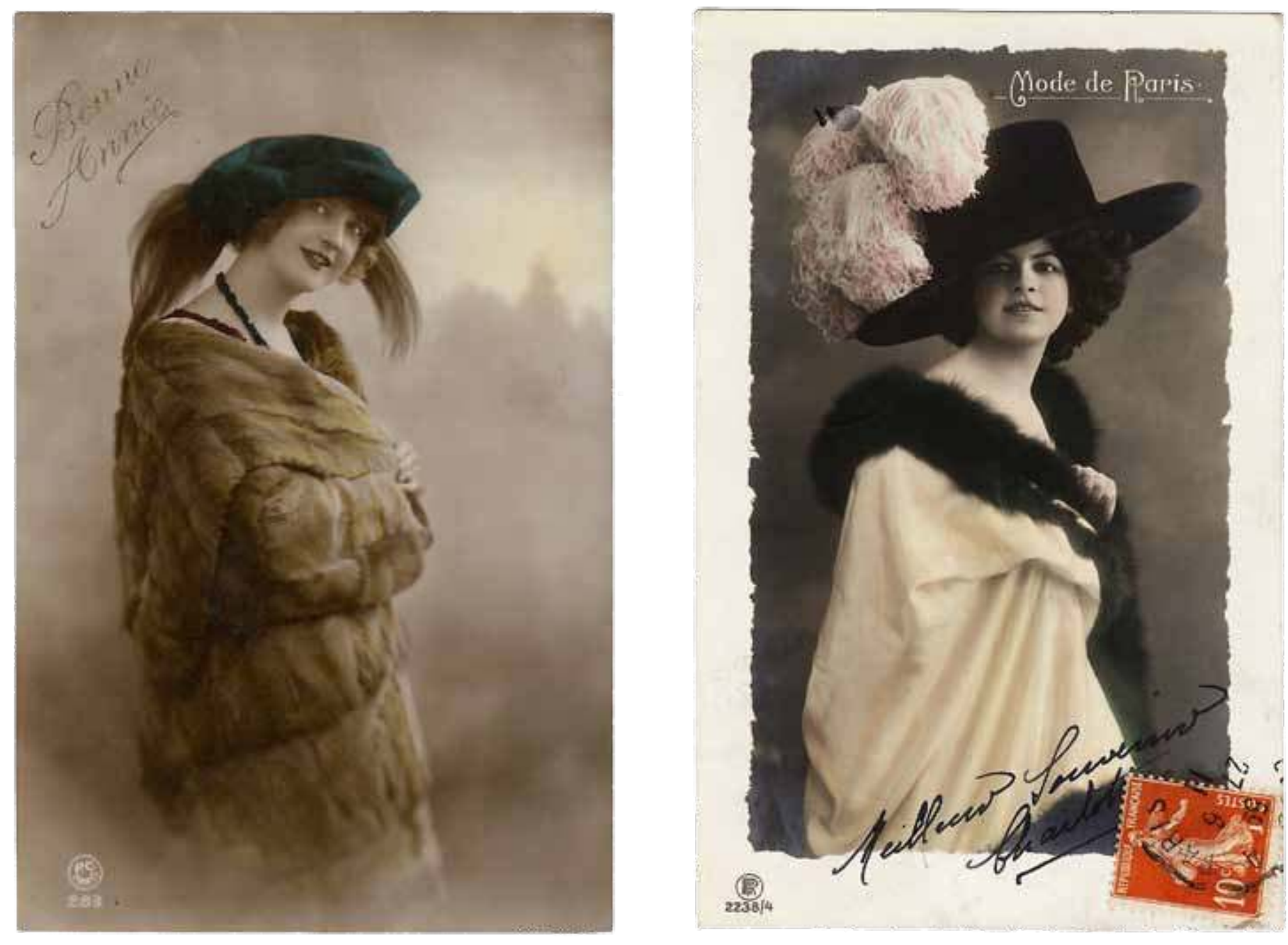

\section{Les codes de convenance}

Autres moyens de diffusion, les cartes postales (coll. Anne Monjaret): elles nous montrent combien en 1900 les parures de plume, en paradisier ou en autruche, sont prisées. Là encore, l'élégance est à son comble, la plume est un signe distinctif. D'après les codes de convenance diffusés dans les journaux de mode, elle n'est portée, dans les bonnes familles, que par les épouses (Knibiehler et al. 1983: 97-98). Peut-être est-ce parce qu'elle symbolise une certaine frivolité animale qui donne accès à la sexualité? D'un côté, on cache le cheveu, symbole de la sexualité, avec un chapeau et de l'autre, on pare le chapeau d'un motif en plume sensuel, quand ce ne sont pas les cheveux qui sont parés. La pudeur a ses codes, ses espaces-temps privés/publics qui jouent avec le charme de la femme jeune mais mature. En définitive, ce sont aussi les jeunes célibataires qui portent ces chapeaux en plume. Les cartes postales en sont une belle illustration.

(Fig. 4 et 5) "oiseau de Paradis" empaillé, s'étalât sur le devant des turbans et des toques de salon ». Il poursuit en signalant qu'en 1828 « quelques chapeaux habillés portaient "deux oiseaux de Paradis, placés bec à bec, les plumes retombant sur les oreilles" » (Lefèvre 1914: 21) ou qu'en août 1865, « les ornements [...] consistaient en petits oiseaux aux ailes déployées, ou même en simples têtes d'oiseaux posés sur de la gaze ou du tulle » (Lefèvre 1914 : 23). Déjà nous percevons dans ces extraits l'étonnement des auteurs quant à cette pratique qui veut que le chapeau, de préférence l'hiver, s'orne de dépouilles ou de plumes d'oiseaux: aigrettes, colibris, paradis, aigles, hérons, moineaux, pinsons, etc. (Fig. 4 et 5). D'autres auteurs à la verve imagée sont plus critiques. En 1914, Edmond Lefèvre rapporte, non sans arrière-pensée, les propos d' « un chroniqueur indigné », datés de 1865 :

« La mode qui, jusqu'ici s'était montrée sous les dehors d'une capricieuse déesse, ambitionne décidément le titre de divinité cruelle. Après avoir constellé d'acier les cotillons de ses adeptes, retroussé leurs jupons en manière de baldaquin et rangé les végétaux dans l'arsenal de leurs parures, 

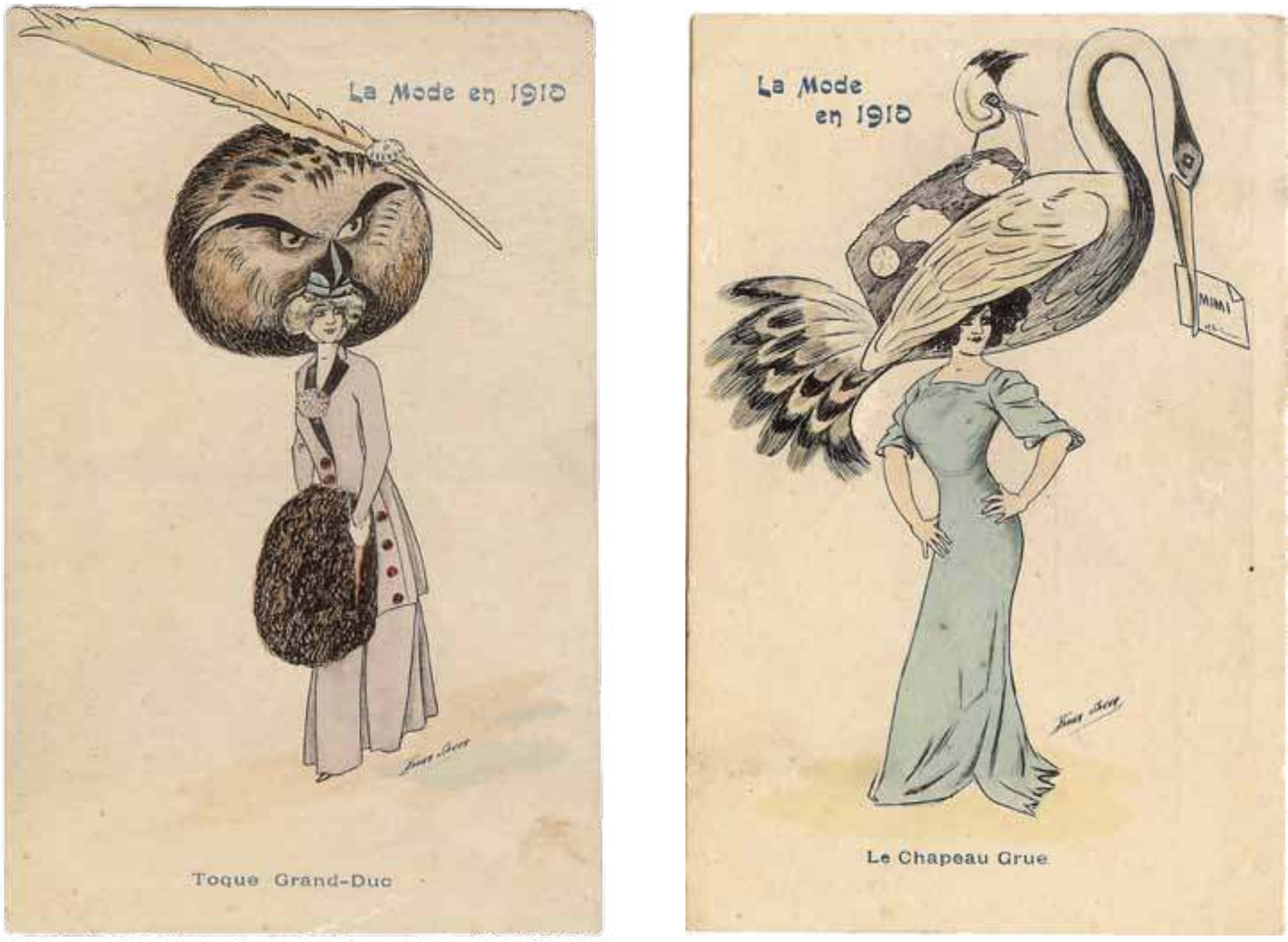

ne s'avise-t-elle pas de coller aux chapeaux des femmes, la tête, voire même le corps des innocents oiseaux du ciel! Si les dames enfiévrées d'innovations s'étaient bornées à s'attifer d'animaux nuisibles, je n'élèverais pas la voix, et je prodiguerais même des encouragements à celles que je croiserais sur ma route, le chef accidenté de punaises ou le front paré de hannetons [...]. Mais quand je vois immoler sur les autels de l'élégance les gracieuses fauvettes et les rossignols mélodieux, je me fâche tout rouge et je demande que l'autorité s'oppose à ce carnage ».

Les ornements des élégantes ne font pas l'unanimité, ils dérangent (Fig. 6 et 7). Est-ce le fait que les femmes s'exhibent dans la rue avec des dépouilles sur la tête ou est-ce leurs attitudes ostentatoires qui offusquent certains? Mais pour Edmond Lefèvre, il n'y a pas de quoi s'inquiéter. Il commente, d'ailleurs avec ironie, la position du chroniqueur dont il a relaté les propos: «Et c'est ainsi que, il y aura bientôt cinquante ans, les protectionnistes modernes avaient déjà un précurseur au style fleuri et à l'esprit plein d'humour et aussi d'amertume » (Lefèvre 1914: 23). Ce défenseur de la nature poursuit, semble-t-il, le débat et même en donne le ton.
Quand les caricaturistes s'emparent de la polémique

Cette mode de la plume est aussi critiquée, les papiers d'humeur des journalistes ne manquent pas ainsi que les dessins des caricaturistes qui s'emparent de la polémique et jouent sur les cordes sensibles du

consommateur. Les deux cartes postales (coll. Bernard Delmas) que nous avons choisies ici sont représentatives de ce jeu de la dérision. Les femmes portent sur leur tête des oiseaux qui se parent eux-mêmes de plume, quand ils ne portent pas leur progéniture sur le dos. Plus encore, le caricaturiste utilise les symboles manipulant leurs sens propre et figuré: la « grue » désigne une prostituée.

(Fig. 6 et 7 ) 


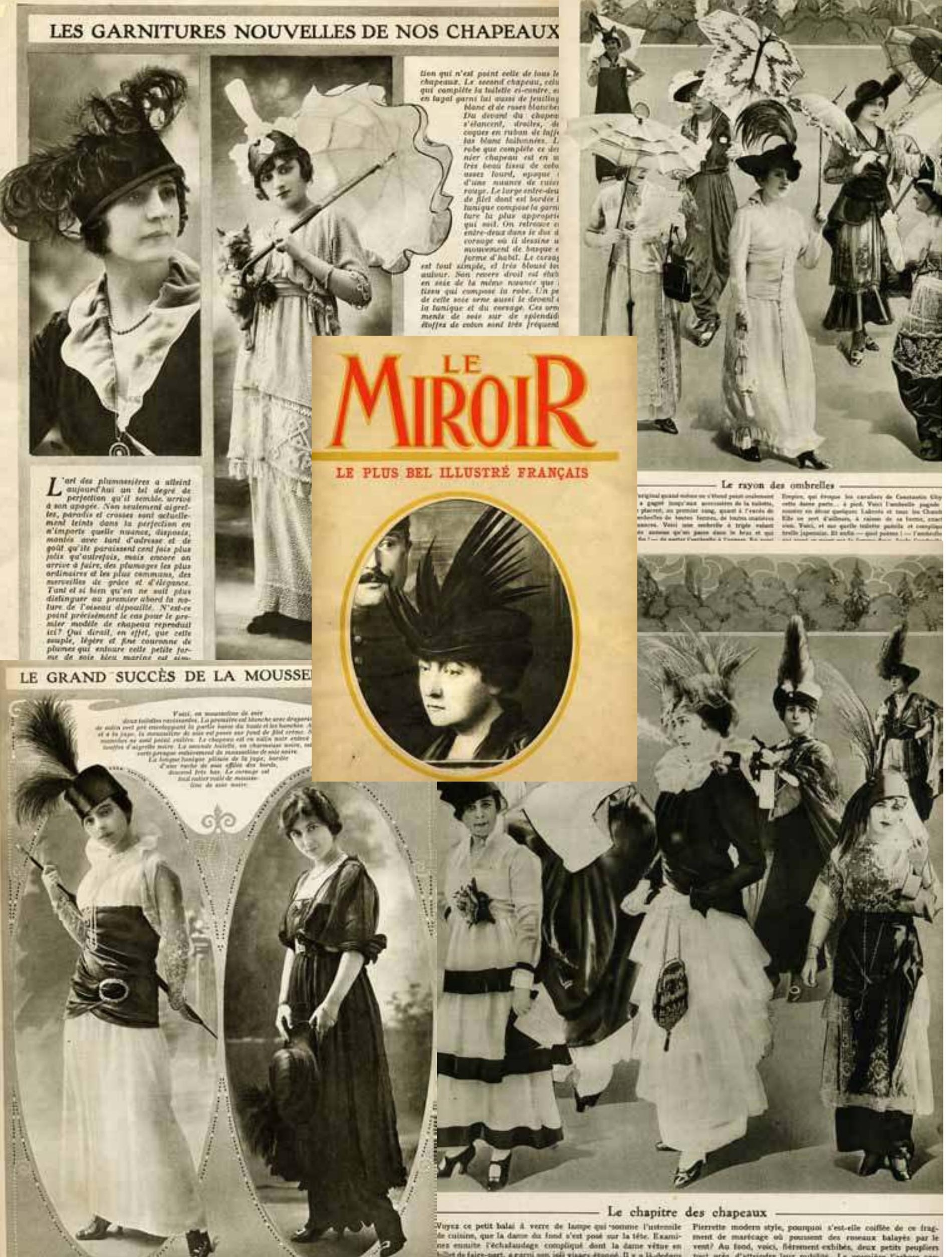


Il semble, néanmoins, que la critique vise plutôt les professionnels de la mode - plumassiers (industriels et artisans) ou modistes -, faiseurs de trouble moral, que les femmes amatrices de belles parures. C'est d'ailleurs au moment où le commerce et l'industrie de la plume de parure se généralisent, au moment où ce secteur économique - porté par la mode et diffusé par la presse (Fig. 8) - devient florissant, que les reproches sur la grande consommation de plumages se font les plus virulents. Détracteurs et défenseurs de la cause plumassière s'affrontent. Il est un fait: la critique n'est pas nouvelle; elle est déjà présente dès le milieu du XIX ${ }^{e}$ siècle.

\section{...aux plumassiers accusés de la destruction des oiseaux : un vieux débat}

Fin du XIX et début du XXe siècle, les auteurs présentant les métiers de la plume argumentent tous contre la destruction des espèces animales, mais les arguments mobilisés diffèrent selon les optiques: les uns incriminent plus ou moins explicitement les plumassiers, les autres les défendent.

Ainsi, Pierre Calmettes dresse un sinistre tableau : en Amérique, en Asie, en Océanie et en Afrique, « les oiseaux, comme les animaux à fourrure, sont décimés par les chasseurs indigènes qui les traquent sans souci de leur disparition possible. C'est ainsi que les aigrettes, le héron aigrette et le héron crosse ou garzette sont détruits par millions chaque année, depuis que les femmes élégantes exigent toutes sur leurs chapeaux, ces petits plumets blancs, légers et mobiles. [...] Et les chasseurs tuent également par millions les lophophores, les satyres, les martins-pêcheurs, les perruches, les argus, les faisans dorés, les oiseaux de velours, les sifilets, les colibris, les nicobars, les marabouts, les lacopodes et les paradisiers au merveilleux plumage » (Calmettes s.d. : 224-225). Lauteur ne mâche pas ses mots et rejoint un public sensible à la cause animale qui reproche aux plumassiers de se fournir en plumage d'espèces exotiques ou rares et de stimuler de la sorte le jeu de l'offre et de la demande et par là même le massacre de ces oiseaux. Rappelons qu'à cette époque, l'économie coloniale pousse à l'exploitation à outrance des ressources locales.

Dans un tel contexte - la critique est vive et publique -, les plumassiers vont être contraints de se positionner, de répondre aux attaques en trouvant des arguments à la fois rassurants et persuasifs. Ainsi, en 1912, la société anonyme Sciama, fournisseur de plumes, écrit dans une publication sortie à l'occasion de son centenaire: «Que le public se rassure! On ne tue plus d'oiseaux indigènes, ni en Europe, ni aux États-Unis pour la fabrication des plumes de parure. Si quelque partie de leur plumage est parfois employée, c'est que l'oiseau a été chassé auparavant pour l'alimentation... » (Sciama 1912 : 60). Si cette démarche informative s'avère insuffisante pour apaiser les esprits des protecteurs de la cause animale dont certains journalistes ou écrivains se font les porte-parole, elle contient en revanche des arguments auxquels les naturalistes ne sont pas insensibles. La Société Anonyme Sciama fait ainsi valoir ses liens avec les ornithologues du Muséum d'histoire naturelle de Paris (Sciama 1912). Cet appui scientifique apparaît alors comme garant de ses activités.
Ces nouvelles pages de la revue Le Miroir (1914) présentent une mode de l'ostentation. II faut savoir porter pour mieux montrer ces couvrechefs parés. Les élégantes semblent se promener sur un champ de course stylisé. Les modèles de chapeaux sont très variés. La plume reste une garniture à promouvoir. Dans l'un des articles, on peut lire à propos de l'une des photographies présentée ici en haut à gauche un intéressant commentaire de mode qui montre la place de cette garniture dans les productions de l'époque: "Lart des plumassiers a atteint aujourd'hui un tel degré de perfection quill semble arrivé à son apogée. Non seulement aigrettes, paradis et crosses sont actuellement teints dans la perfection en nimporte quelle nuance, disposés, montés avec tant d'adresse et de goût quilis paraissent cent fois plus jolis quáatrefois, mais encore on arrive à faire, des plumages les plus ordinaires et les plus communs, des merveilles de grâce et

d'élégance. Tant et si bien quion ne sait plus distinguer au premier abord la nature de l'oiseau dépouillé. N'est-ce point précisément le cas pour le premier modèle de chapeau reproduit ici? Qui dirait, en effet, que cette souple, légère et fine couronne de plumes qui entourent cette petite forme de soie bleu marine est simplement en plumes de coq, mais combien méticuleusement apprêtées! ». Le travail de transformation du plumassier est souligné avec précision.

(Fig. 8) 


\section{L'association des ornithologues et des plumassiers pour soutenir la mode}

Les plumassiers trouveront en effet dans la voix de ces professionnels, « naturalistes plumassiers » de métier ou amateurs éclairés, les arguments d'une justification, et plus encore de leur légitimation et de leur valorisation. Comment l'alliance des plumassiers et des ornithologues s'énonce-t-elle? Quel type de discours est alors tenu? Et encore une fois, que nous disent ces discours du contexte de l'époque? Car c'est là le noud de l'affaire. Les plumassiers sont contraints de trouver les moyens de légitimer leur production, de montrer leur bonne volonté ; seuls, ils apparaissent démunis.

\section{La justification d'ornithologues pour une légitimation des plumassiers}

Les ornithologues sont en quelque sorte venus à la rescousse des plumassiers et les soutiennent fermement. Dans un article daté de 1898 consacré à « La question des oiseaux. Contribution universelle des oiseaux dans l'industrie des plumes de parure », un certain Jules Forest dit se passionner depuis longtemps pour la question de la « protection aux oiseaux ». Cette passion l'a entraîné « dans de longs et coûteux voyages d'études ». Suite à ses observations, il « démontre combien sont exagérées, sinon contradictoires, les plaintes adressées aux sociétés protectrices des animaux contre l'abus de l'usage des oiseaux de nos contrées et de leur plumage dans la parure humaine » (Forest 1898: 780). Il souhaite « apporter quelques éléments d'appréciation sur le plus ou moins bien fondé d'une opinion qui attribue à tort, à l'industrie plumassière, à la Mode, la cause principale de la disparition, de la destruction de nombreux oiseaux plus ou moins utiles à l'agriculture » (Ibid. : 779).

Plus tard, en 1914, Edmond Lefèvre va également dans ce sens et prend clairement position pour les plumassiers qu'il considère comme de véritables boucs émissaires alors que ces derniers sont au contraire soucieux de cette faune:

« Malgré les nombreuses réclamations adressées aux pouvoirs publics, rien n'a été fait jusqu'ici et on se contente de faire retomber sur le dos des plumassiers la disparition progressive de ces oiseaux, alors que la faute ne leur en est nullement imputable. [...] Il est évidemment d'importance capitale pour les plumassiers de voir cesser, au plus tôt, cet état de choses, qui tend à diminuer de plus en plus leur champ d'action et apporte une gêne considérable dans l'exercice de leur profession » (Ibid. : 324).

Pour lui: « les intérêts des protectionnistes et des plumassiers ne sont nullement incompatibles; en effet, les commerçants n'ont aucun intérêt à voir disparaître les oiseaux, et il est absolument certain que si le trafic des plumes était fait d'une façon rationnelle, il n'y aurait à craindre ni la disparition des espèces, ni même leur diminution » (Lefèvre 1914 : 326). 


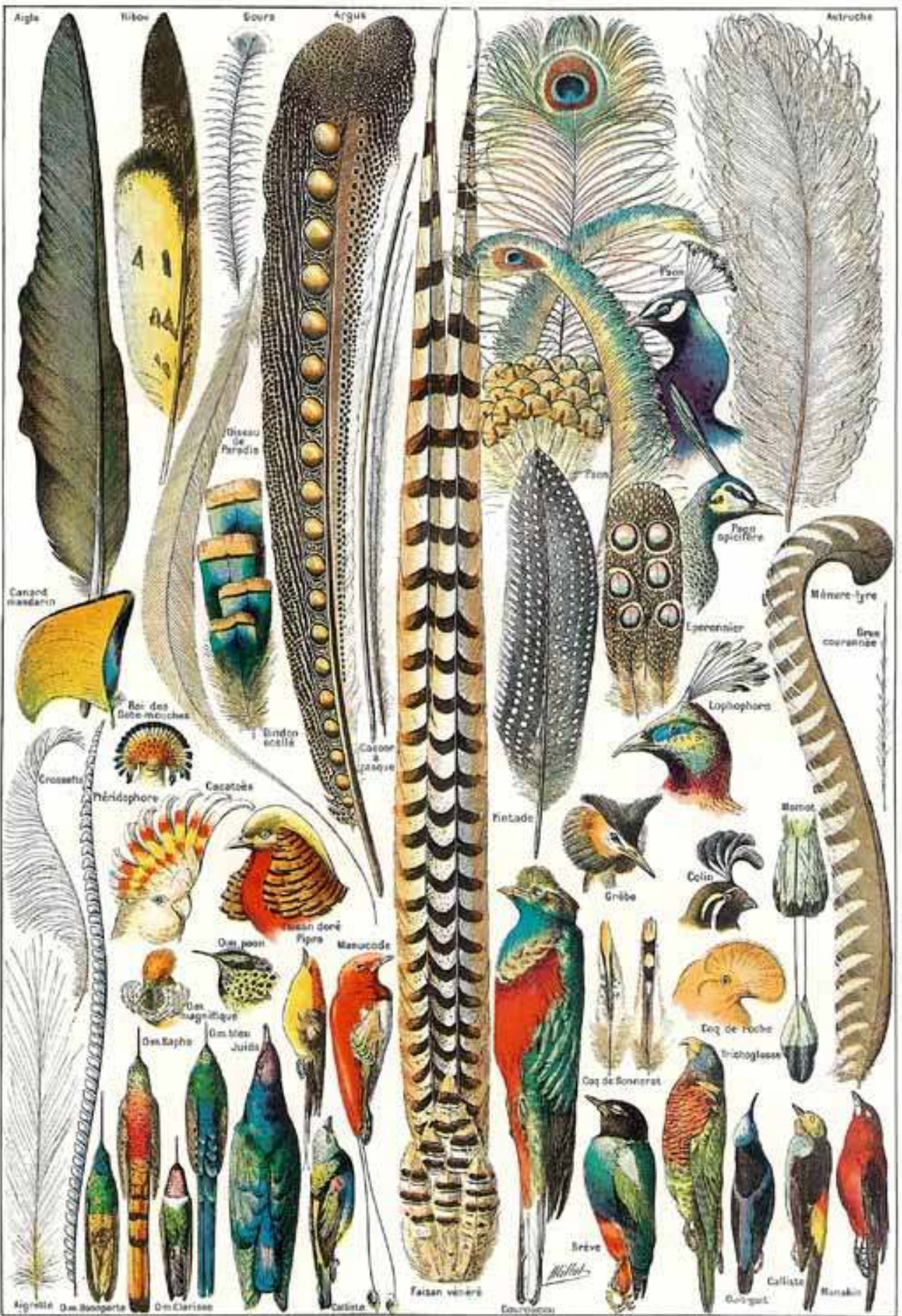

des ornithologues

Cette planche tirée du

Larousse Ménager de

1926 est l'illustration

qui accompagne la

notice « plume ». Elle

ressemble à une

planche scientifique

d'ornithologues en

présentant une variété

d'espèces (oiseaux,

plumes ou de têtes),

mais ici la spécificité,

cest que ces plumes

sont utilisées par

l'industrie plumassière

de la parure.

(Fig. 9) 
En 1908, cette alliance entre les plumassiers et certains ornithologues sera confirmée par la création d'un comité de défense des industries plumassières. Son but est de servir les intérêts des professionnels de la plume pour parure. En 1911, ce comité se transforme sur les conseils de M. H. Blumenfeld-Sciama en commission intersyndicale et deviendra par la suite le comité d'ornithologie économique dont fait partie Edmond Lefèvre. Les membres, très actifs, se sont donné pour objectif de répondre avec fermeté aux articles considérés comme tendancieux. Pour Léon Bollak (1914) par exemple, l'emploi des plumes d'oiseaux sauvages peut se faire sans menacer ces espèces. De la même manière, Edmond Lefèvre s'en prend aux inepties des chroniqueurs qui ont écrit que l'on scalpait les aigrettes vivantes alors que ces oiseaux portent leurs plumes non sur la tête mais sur le dos ${ }^{4}$, ainsi qu'aux sociétés s'occupant de zoologie et d'ornithologie qui ont associé à tort ces usages « exclusivement à la consommation toujours croissante de plumes faites par l'industrie de la mode » (Lefèvre 1914 : 324).

\section{Les « véritables fautifs »}

Déployant des arguments à foison pour contrecarrer la critique et sortir les détracteurs de leur ignorance, les défenseurs de la cause plumassière désignent ceux qui sont à leurs yeux les « véritables » auteurs de la destruction des oiseaux dans le monde.

Ainsi, dès 1898, Jules Forest souligne que «Chaque année, chaque saison, ce sont modes nouvelles; alternativement la répercussion de la mode se porte sur les oiseaux les plus disparates, sans considération d'oiseaux utiles ou nuisibles ». Ce renouvellement perpétuel implique argumente-t-il - le prélèvement annuel des espèces de toutes sortes et de toutes provenances. Pour la mode, il « n'est qu'une cause occasionnelle du massacre [...] la chasse par sport et pour l'alimentation sont des faits bien autrement considérables »; autrement dit, l'auteur cherche à relativiser la part de la mode dans le processus de destruction de la faune ornithologique. À propos de la France, il dénonce aussi les « chasseurs légaux», auxquels « il faut ajouter les propriétaires, les enfants surtout, qui tirent dans les jardins, et surtout enfin une innombrable armée de braconniers pratiquant l'extermination » (Forest 1898: 781), non réprimée par les gendarmes. De surcroît, cette chasse n'approvisionne pas le marché des plumes de la mode qui proviennent surtout des oiseaux d'élevage, de basse-cour, fournis par les Halles de Paris et par les marchands de volailles. Jules Forest se sert d'une note de la chambre syndicale des fleurs et des plumes, datée d'avril 1898, adressée par le bureau de la section de zoologie du congrès des sociétés savantes et par le président de la société nationale d'acclimatation aux départements intéressés pour conforter son argumentation: "Pour la mode et la parure, on n'emploie presque plus d'oiseaux de France » (Forest 1898: 785). Faut-il comprendre que les matières premières de ce secteur d'activité proviennent des chasses lointaines et des productions exotiques, de la variété innombrable des espèces d'autres pays? Même dans ce cas de figure, l'auteur montre que 
la part du marché réservée à la mode française reste modeste au regard du marché international, que l'élevage et la domestication des oiseaux sont des réponses innovantes aux besoins des plumassiers et respectueuses de la faune. Le parti pris est clairement énoncé.

Plus tard, en 1914, Edmond Lefèvre, s'il rejoint dans le fond Jules Forest, mobilise d'autres arguments et nuance pour sa part la responsabilité exclusive des chasseurs dans la disparition totale ou partielle de certaines espèces d'oiseaux. Selon lui, d'autres facteurs « dont les effets néfastes se font sentir depuis fort longtemps » sont à prendre en considération: le défrichement et l'envahissement progressifs par l'homme de régions qui servent d'habitat à un grand nombre d'espèces; le développement des moyens de transport qui permettent aux chasseurs de se rendre rapidement sur les lieux de chasse; le perfectionnement des armes; la définition de la chasse comme un sport (Lefèvre 1914 : 321). Il ajoute également que « bien avant que n'existassent les causes de cette destruction dues aux progrès de la civilisation, les peuples plus ou moins sauvages avaient commencé à tuer un grand nombre d'oiseaux de leurs propres pays pour se procurer leurs plumes, qu'ils employaient à la parure ou à divers autres usages » (Lefèvre 1914 : 322). Citant l'exemple de la Chine, de l'Australie et de l'Amérique (tribus indiennes), l'auteur dédouane de cette façon les Européens. Ce type de discours daté est à recontextualiser comme l'expression idéologique de la suprématie européenne sur les cultures lointaines qualifiées de « sauvages ». Il sous-entend en outre que ces pratiques ont toujours existé, car elles sont en quelque sorte le propre de l'homme. Enfin, il n'hésite pas non plus à revenir sur le cas français pour mieux déresponsabiliser, disculper encore les plumassiers. Les « tueurs » se rencontrent également dans nos contrées et Edmond Lefèvre considère qu'il existe des « tolérances inexplicables pour la capture des oiseaux au moyen de filets de toutes sortes, d'appeaux, etc. Ces choses-là qui se font avec le consentement tacite des gardes champêtres et de la gendarmerie. [...] Ce n'est qu'en France qu'on tolère ces destructions, car dans les pays voisins on sait faire respecter la loi très sévèrement » (Lefèvre 1914 : 323). Largumentaire de ce « défenseur de la nature » tient de leçon de morale. Vrais, faux ou imprécis, ce n'est pas la véracité des propos qui nous intéresse ici, mais l'analyse d'une rhétorique, servant avec ferveur à asseoir son plaidoyer.

Grâce à un discours savamment mené, fine construction idéologique, souvent documenté (exemples chiffrés et descriptions ethnographiques à l'appui), les auteurs réussissent le tour de force d'affranchir de toute suspicion les plumassiers. De la sorte, les défenseurs de la cause plumassière plaident conjointement en faveur de celle des oiseaux et trouvent a priori un terrain d'entente sur la question sensible de la destruction des espèces ornithologiques, avec les détracteurs de la pratique plumassière.

La conviction des ornithologues sert non seulement l'économie plumassière française confrontée à une rude concurrence étrangère, mais également les intérêts des naturalistes. Lune des tâches du naturaliste est en effet la constitution de collections de spécimens, en l'occurrence ornithologiques ${ }^{5}$, qui implique le prélèvement de la faune dans son environnement naturel 
(Fig. 9). Bien que leurs objectifs professionnels soient différents, naturalistes et plumassiers se trouvent confrontés aux réglementations nationales et internationales de protection animale qui régulent les circulations, les entrées et les sorties d'un pays à un autre.

\section{Des conventions pour la protection animale}

Au XIX ${ }^{e}$ siècle, certains pays commencent à édicter des lois visant à protéger les espèces en voie d'extinction. Si ces lois n'ont pas toujours l'effet escompté, elles amorcent le mouvement des réglementations et sensibilisent à la cause animale les professionnels de ces circuits économiques. Et elles invitent aux débats. C'est en cela que nous pouvons parler d'éveil d'une sensibilité à la vie animale. En 1926 encore, on peut lire dans le Larousse Ménager que « Le massacre de certains de ces oiseaux pour parure a été si grand que les gouvernements des pays d'origine ont dû intervenir pour les protéger et empêcher la destruction de l'espèce » (Larousse Ménager 1926 : 952). Ces propos laissent entendre que les États sont donc bien conscients des excès de ces pratiques et qu'ils interviennent pour les limiter.

Cependant, à y regarder de près, certains paradoxes apparaissent. Ces lois, loin de ne servir que la cause animale, peuvent en effet être soustendues par des intérêts économiques et protectionnistes pour contrôler les marchés, les importations et les exportations. Il faut les resituer dans le contexte concurrentiel des marchés internationaux de l'époque et du commerce des comptoirs coloniaux florissant. Ces dispositions législatives ont eu des conséquences directes ou indirectes sur l'économie plumassière de la Mode. Mais quelles sont les directives de ces lois?

\section{La plume sous les griffes de la loi et la riposte de groupes de pression}

Les défenseurs de la cause plumassière sont des lecteurs éclairés et des commentateurs virulents de ces lois. Dès 1898, Jules Forest signale une loi adoptée par les Américains, « prohibant l'importation, la transportation et la vente à l'intérieur du territoire américain de toutes les peaux et parties de peaux et plumes d'une série d'oiseaux chanteurs ». Son but est - justifie un autre auteur - " de protéger ces oiseaux contre le massacre "stupide" qui s'est fait sans cesse, à l'effet de fournir des plumes aux modistes pour les chapeaux féminins ». Ce même auteur renchérit : "Quand on prendra une mesure analogue en France, nous en serons fort heureux ». Jules Forest réagit à cette leçon de morale qu'il considère «tendancieuse » :

« Je ne saurais trop protester contre de telles assertions provenant certainement de personnes fort bien intentionnées à l'égard du monde ailé, et par ignorance, fort malveillantes à l'égard de la corporation des naturalistes plumassiers, dont j'ai l'honneur de faire partie. Je ne crois pas exagérer en affirmant ici que la plumasserie n'emploie pas d'oiseaux chanteurs 
européens; en général leur petite taille ne permet pas leur utilisation taxidermique trop coûteuse. C'est pour l'alimentation que se fait la chasse des alouettes, des mauviettes, des grives, des merles, oiseaux chanteurs de nos pays » (Forest 1898: 784).

À travers ces propos, nous percevons le ton et le contenu des débats d'alors entre les partisans et les détracteurs de l'industrie et de l'artisanat plumassiers. Pour ce naturaliste, la critique ne vaut pas pour les productions des modistes. Il n'hésite pas à faire valoir que ces professionnels de la parure recherchent peu les insectivores aux plumages trop neutres et qu'il leur est possible de toutes les façons, de les remplacer par des nuisibles.

Edmond Lefèvre évoque, de son côté, le fait que des conventions internationales ont été signées au cours des premières décennies du $\mathrm{XX}^{\mathrm{e}}$ siècle pour limiter les « tueries»; il souligne toutefois les difficultés de leur application ou les ripostes internationales qui ont suivi. Étonnamment, il ne mentionne pas la première grande convention signée à Paris en 1902 qui concerne la protection des oiseaux « utiles à l'agriculture » et qui, entre autres choses, prohibe les moyens de captures tels que les pièges, cages, filets, lacets et gluaux. Edmond Lefèvre s'arrête sur un projet de loi anglais daté de 1908, qui vise à interdire l'importation des plumes et des peaux d'oiseaux exotiques. Les réactions en Angleterre et en France ne se font pas attendre. Rien n'y fait. D'autres projets analogues seront présentés en 1909. En 1913, face à une violente campagne menée dans différents pays pour obtenir la prohibition absolue de certaines catégories de plumes, les États-Unis sont conduits à la mise en application d'une loi interdisant l'introduction et l'emploi des plumes de tous les oiseaux sauvages (Lefèvre 1914 : 325). La France n'adhère pas à cette proposition qui met en danger un secteur de production qui emploie de nombreux ouvriers et ouvrières. Le dirigeant d'une des maisons les plus réputées sur la place de Paris considère que cette convention américaine de 1913 connaît des « anomalies » : elle stipulerait l'interdiction d'utiliser des plumes d'oiseaux qui ne se mangeraient pas sur place. Pourtant, le faisan de Chine consommé en Chine est cependant interdit à la mode; en revanche, un contingent est accepté pour la fabrication des mouches artificielles destinées à la pêche. D’autres lois prendront le relais ${ }^{6}$.

\section{La convention internationale de Washington, des conséquences toutes relatives pour la mode}

Adoptée en 1973, la convention internationale de Washington (CITES) entre en vigueur en 1975 et réglemente depuis la circulation des espèces menacées d'extinction, spécimens vivants comme morts, dont les oiseaux qui sont ou pourraient être affectés par le commerce. La France la ratifie en 1977 et la met en place en 1978. Des listes d'oiseaux de divers pays d'origine apparaissent dans les annexes I, II et III. Nous n'en dresserons pas la liste exhaustive, mais en retiendrons quelques-uns: certaines espèces de nandous (Rheidae), les grèbes (Podicipedidae), les albatros (Diomedeidae), les pélicans (Pelecanidae), les frégates (Fregatidae), les cigognes (Ciconiidae), 
l'Environnement et la CITES qui délivrent des certificats. Les conditions de surveillance obligent les professionnels à déclarer les mouvements de leurs produits et à s'armer de certificats phytosanitaires pour toute exportation (le certificat du fournisseur de plumes s'ajoute alors à celui du créateur). Certains artisans de luxe usent de matières premières d'origine animale en raréfaction, prélevées dans leurs vieux stocks. S'ils ne respectent pas les règlements de circulation des oiseaux, ils savent qu'ils peuvent tomber sous le coup de la loi. Un plumassier nous rapporte qu'il est actuellement possible de sortir des pièces de vêtements ornées de plumes, et surtout de les faire rentrer aux États-Unis munies d'un certificat d'admission temporaire. Une plumassière quant à elle se rappelle des ruses employées pour contourner la loi: " parfois des oiseaux ou des chapeaux en plume, bien enveloppés, passaient la douane sans problème, les douaniers ne savaient pas reconnaître les espèces ». Un grossiste parle sans détour des modes de violation de la loi et se souvient par exemple des marins américains qui faisaient du trafic de plumes et « pour que cela pèse plus, ils y glissaient des épingles ».

Les plumassiers attentifs à toutes ces réglementations n'hésitent d'ailleurs pas à les critiquer, notifiant les limites de certaines mesures et encourageant parfois à la mise en place d'autres mesures plus favorables à leur commerce.

Dès le XIX ${ }^{e}$ siècle, la route des plumes principalement exotiques, sauvages, pour la mode, semble donc avoir navigué entre légalité et illégalité, entre contrôle commercial et surveillance sanitaire. Les plumassiers, soutenus par certains naturalistes tenant des discours légalistes à propos de leurs pratiques semblent s'être rapidement accommodés de ces dispositions juridiques.

\section{Les plumassiers, « écologistes 》: expériences mondiales et initiatives locales}

Les plumassiers, en effet, font preuve d'une capacité d'adaptation aux contraintes non seulement législatives mais aussi économiques, politiques et sociales. Comment s'en sortent-ils? Que nous disent leurs pratiques de leur rapport à la nature?

\section{Du sauvage chassé à l'élevage}

Les plumes exotiques, sauvages, ont été très vite accompagnées, voire supplantées par les plumes d'élevage ou de basse-cour (autruche, nandou, coq, dinde, oie, faisan). Ces dernières ont rencontré un franc succès auprès des plumassiers et artisans de la parure qui ont vu là une matière économique et malléable. Grâce à une connaissance ornithologique poussée, la récolte de plumages peut se faire sans atteindre l'animal sauvage (Fig. 10). Les plumes de l'aigrette se récupèrent après la mue. Laviculture est une autre réponse écologique qui pallie aux « massacres » des oiseaux 
et une réponse commerciale qui contribue à la maîtrise et au rendement de la production, réduisant la dimension aléatoire de la chasse et évitant le geste de capture (Pujol \& Carbone 1990: 1347). Pourtant, tous les oiseaux ne sont pas domesticables. Il en est ainsi des paradisiers très prisés des plumassiers de mode.

« Il est regrettable, pour la conservation de ces oiseaux aux plumes étincelantes, qu'ils soient rebelles à la domestication, et qu'ils ne puissent s'élever dans les fermes, comme on élève les autruches au Cap, en Égypte, en Australie, en Californie et au Texas » (Calmettes s.d. : 225).

Les élevages ne suppriment donc pas complètement la question de la protection des espèces animales en voie de disparition. Les premières tentatives, en particulier d'élevage d'autruches, considérées par certains comme un progrès incontestable, seraient datées des années 1866-1870 (Coffignon s.d.; Sciama 1912).

« Depuis une quinzaine d'années, des essais de domesticité ont été tentés dans quelques pays et les résultats en Égypte, et surtout dans la colonie du Cap, sont très importants, bien que la perfection des produits reste au-dessous du plumage des oiseaux sauvages qui n'existent pour ainsi dire plus maintenant » (Lami 1887: 421).

L'émergence de ce nouveau marché provoque inévitablement une concurrence internationale que les plumassiers français auraient sousestimée au départ (Larousse Ménager 1926 : 951). Des producteurs se sont malgré tout lancés, dès 1879, dans des élevages d'autruche en Algérie, alors colonie française. Certaines tentatives se sont soldées par de coûteux échecs, comme le rapporte le jury de l'Exposition universelle internationale de 1900 à Paris. Alfred Franklin évoque en 1896 néanmoins l'arrivage de plumes d'autruche venant d'Alger et plus généralement de l'Afrique qui a été et reste un continent fournisseur majeur. En 1912, la société anonyme Sciama souligne les actions qu'elle a entreprises en faveur de l'élevage, qu'elle encourage et valorise. En France, des tentatives d'élevage d'autres espèces ont existé: celles de nandous en Seine-et-Marne, de lophophores dans les Ardennes ou bien encore de faisans versicolores et de dindons blancs dans d'autres régions. Des héronneries sont également mentionnées au Venezuela, des élevages de dindons blancs à l'ouest des États-Unis et en Hongrie (Sciama 1912). La domestication permet de prélever à la mue, « tous les neuf mois », les plumes de l'animal, sans avoir à le tuer. Lanimal à plume se distingue ici de l'animal à poil. Les plumes des ailes, et précisément celles des mâles adultes, jugées plus belles parce que colorées, sont les plus prisées pour travailler les parures.

En développant progressivement de nouveaux marchés, en particulier de plumes d'élevage, les plumassiers ont su se maintenir sur la place commerciale mondiale et valoriser leurs productions avec des techniques de retraitement de cette matière première. Ils ont su exercer une maîtrise sur la « nature » en la dressant, en la transformant pour mettre l'accent encore davantage sur son artificialité. 


\section{Faire plus vrai que nature: une recherche d'authentique avec du vrai-faux}

Le travail de transformation des plumes, exotiques ou domestiques, est au coeur de l'ouvrage des artisans plumassiers. C'est dans ce travail créatif que ces hommes de métier trouvent le moyen d'épargner les oiseaux sauvages et d'adhérer aux conventions pour la défense de la faune. Leur art tient à une recherche des effets, à une capacité de transformer la matière première, de la remodeler, pour jouer sur son apparence, en faire un autre objet, une marchandise toujours proche de la nature mais différente de sa forme initiale (Celnart 1838; Franklin 1905-1906; Lefèvre 1914).

La plume exotique reste emblématique mais chère, et sa circulation très réglementée influe sur les conditions de production. La plume domestique, quant à elle, est bon marché. La diversité des plumes et le fait qu'elles ne soient pas soumises aux contraintes et interdits législatifs contribuent à un jeu de substitution. Limitation devient alors un atout écologique et économique: " avec les plumes de dinde ou d'oie, on imite le marabout, avec du coq ou du nandou, on fait du paradis, l'oie fournit de jolies crosses, les paons, de belles aigrettes » (Larousse Ménager 1926 : 952). De même, les plumes d'oie, de faisan, de paon ou celles d'autruche passées dans un bain d'acide trouvent les qualités visuelles, les aspects de l'aigrette ou du paradis interdits à la circulation.

Le travail consiste donc à rester au plus près de la nature tout en changeant ce naturel grâce à des artifices techniques (Fig. 11). Les plumes blanchies puis teintées, frisées et coupées miment les plumes exotiques et confèrent de la variété à la production (Forest 1898; Exposition universelle 1900). Grâce au goût et à l'ingéniosité de l'artisan, la plume ordinaire peut être métamorphosée en une plume venue d'ailleurs. La qualité et les « tournures élégantes » de ces métamorphoses font la réputation internationale des ouvrières parisiennes et représentent un atout important pour lutter contre la concurrence, tout particulièrement anglaise et allemande (Lami 1887: 420; Exposition universelle 1900 : 366). C'est ainsi qu'un véritable marché de fausses parures se développe.

Les dépouilles (tête, aile ou oiseau en entier dont le corps est rempli d'herbes) prisées à la Belle Époque sont retravaillées pour donner forme à un nouvel oiseau avec « les plumes les plus étranges, composées à l'aide des dépouilles d'oiseaux de toutes couleurs, gouras couronnés, perruches, sifilets, moineaux même, moineaux à la livrée grise ». Les oiseaux sont « transformés en oiseaux multicolores, en parures luxueuses. [...] Et les paradis eux-mêmes sont truqués. Valant en gros cinquante francs pièce, les marchands détaillants veulent en tirer le meilleur parti possible, et, dépioté dès son arrivée, déchiqueté plume à plume, un paradis en formera parfois cinq ou six après le recollage des plumes. Les têtes et les ailes servent à d'autres garnitures » (Calmettes s.d. : 229). Ce que certains qualifient de tricherie et de supercherie peut apparaître pour d'autres comme un acte de protection, de respect de la nature. Pour Pierre 


\section{Des plumes en fleur}

Déjà au XVIII' siècle, comme nous le montre cette planche tirée de L'Encyclopédie de Diderot et d'Alembert, la plume est travaillée pour se métamorphoser en autant de formes possibles. Ainsi, la plume rencontre la fleur, se transforme en un autre produit de la nature. C'est au cours du XVIIII siècle que les plumassiers se mirent à travailler les fleurs de fantaisie à l'aide de plumes ou d'étoffes. En 1776, avec la réorganisation des communautés de métiers, ils prendront d'ailleurs la qualification de « plumassiersfleuristes » et partageront le privilège du travail de la plume et des fleurs artificielles avec les marchandes de modes, encore appelées « faiseuses de mode », qui se séparèrent quant à elles à ce moment-là des merciers. À partir de la fin du XIXe siècle, plusieurs profils professionnels se rencontrent: des artisans et commerçants qui cumulent les activités de plumassier et de fleuriste, d'autres qui se spécialisent dans l'une ou l'autre des productions. Les rythmes de ces deux industries restent toutefois indissociables: la production des fleurs a lieu en hiver pour la mode de l'été et celle des parures en plumes assurée le plus souvent par les mêmes ouvrières, a lieu en été pour les collections de l'hiver.

(Fig. 11)

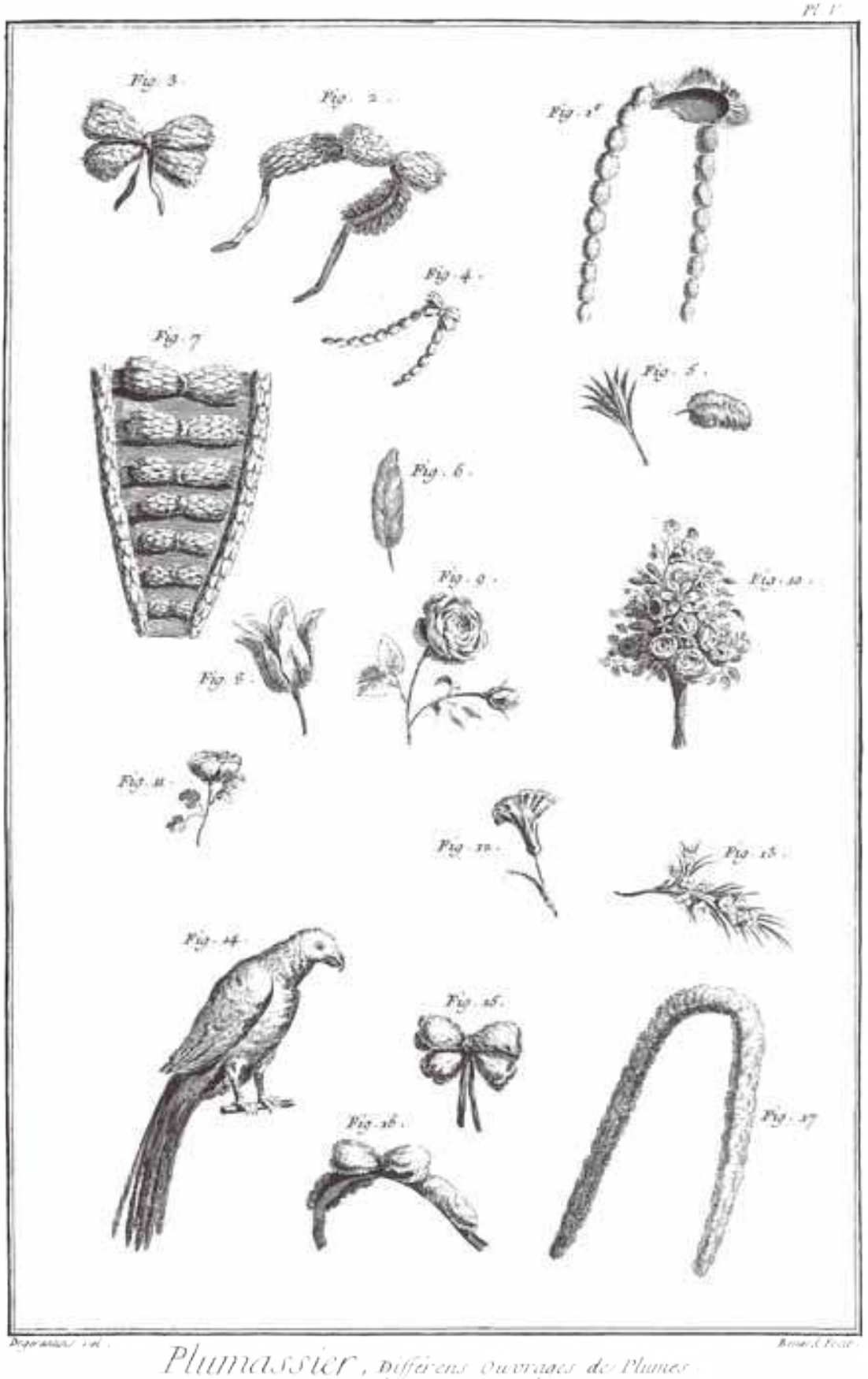


Calmettes, toutes ces initiatives ne changent rien aux « massacres », et n'empêchent aucunement l'atteinte à l'intégrité animale:

« les fabricants de fausse parure trouvant avantageux de copier les aigrettes avec des plumes de paon et autres oiseaux rares avec des plumes de poules, de dindes et d'oiseaux d'eau: mouettes, macareux, pétrels, pingouins, canards, etc., tués chaque année par millions dans les Îles du Pacifiques et les régions septentrionales » (Calmettes s.d. : 225).

Le port sur un chapeau d'une dépouille pose problème, et s'afficher de la sorte relèverait désormais de la provocation. Rien ne doit rappeler l'animal authentique, vivant ou mort. On assiste en quelque sorte à l'« éviction de l'animal » (Fourmaux 1992 : 21-24). Lartifice crée une distance supplémentaire avec le réel en chair et en plume (Fig. 12). Laspect naturel de la plume, nous dit Francine Fourmaux, n'est surtout pas valorisé, il est modifié voire effacé " au profit d'une œuvre humaine, civilisée » (1992 : 24). Comme le dit un plumassier, encore aujourd'hui, et surtout aujourd'hui: " on rechigne à mettre des oiseaux naturels. On fait des oiseaux recréés » (Fig. 13). Cela s'explique-t-il par le fait que les clientes refusent désormais de paraître en public avec une dépouille sur la tête, craignant le ridicule ou la vindicte des écologistes, ou par le fait que les plumassiers entretiennent de nouveaux rapports à l'animal? On le sait, nous sommes de plus en plus sensibles à la cause animale, mais c'est sans doute dans les ateliers, en interrogeant les plumassiers qui travaillent cette matière que nous trouverons la réponse. Comment ces artisans vivent-ils ce contact à l'animal, à sa dépouille au moment de sa transformation? Comment opèrent-ils pour l'accepter? Francine Fourmaux nous apporte une piste intéressante: alors que les plumes sont considérées du point de vue zoologique comme des croissances mortes, elles sont prises par ces mêmes artisans pour une matière vivante (Fourmaux 1992 : 13) qu'ils retravaillent, un peu à la manière d'une chevelure.

Lart du plumassier, qui n'est pas le traitement de l'oiseau naturalisé par le taxidermiste, s'exercerait particulièrement dans la faculté à faire du vrai-faux avec du vrai, c'est-à-dire une autre plume, un autre oiseau, donc à faire de l'authentique et également à faire de l'exotique avec du domestique. Là est la spécificité du plumassier. Il se différencie ainsi du fleuriste en fleurs artificielles qui lui fait du faux en copiant du vrai, fait de la nature, son modèle (Celnart 1838; Picard 1979; Fourmaux, Monjaret 1993). Il est, en revanche, plus proche du fourreur qui, travaillant le poil et la peau, transforme la nature (Fig. 15 et 16). Le plumassier lui emprunte d'ailleurs certains termes. Francine Fourmaux remarque qu'aujourd'hui, les professionnels de la plume cherchent plutôt «à se détacher et se distinguer des fourreurs et des peaussiers, trop en contact avec l'animal luimême ». À aucun moment, ils n'évoquent le cuir et la fourrure de l'oiseau (Fourmaux 1992 : 23). Finalement, le tabou porte sur l'animal et non sur la plume. La recherche du prestige se joue dans la plume, dans la belle plume exotique considérée comme une matière noble et précieuse, et par ceux qui la travaillent et par celles qui le portent (Fig. 14).

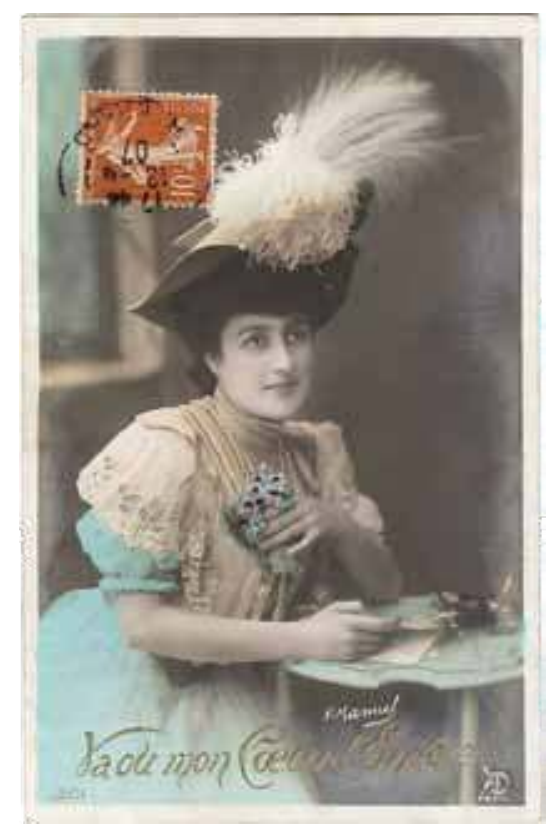

Jouer de l’artifice pour être à la mode

1910

Les espèces se mêlent pour parfaire les ornements à chapeau; l'autruche est combinée à l'aigrette, au paradis (carte postale: coll. Anne Monjaret). Lartisan joue sur la matière et ses aspects, frisant, étirant pour arriver à ses

fins et fabriquer des « fantaisies » qui marquent les tendances d'une année. Chaque motif associe des plumes de provenance différentes; en 1913, certains modèles conjuguent par exemple le paradis et le lophophore (Lefèvre 1914 : 315) qui formeront, ensemble un modèle particulier.

(Fig. 12)

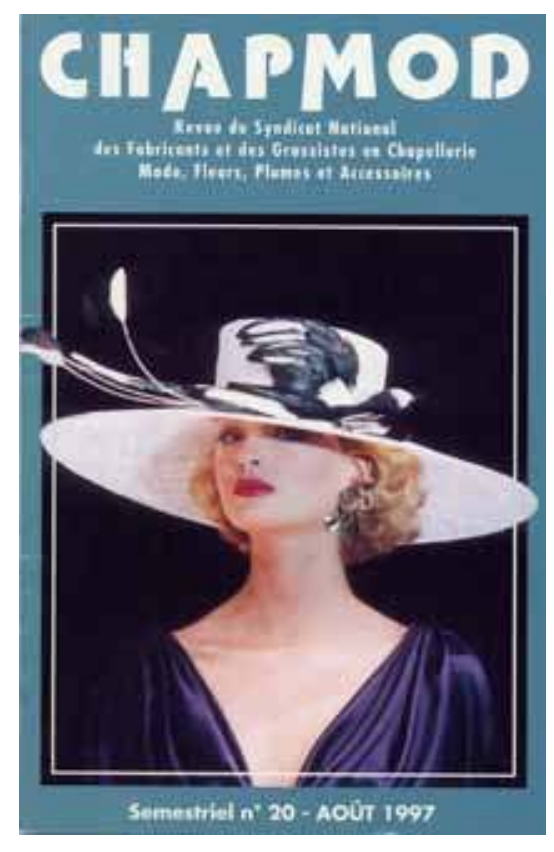

1997

Ce chapeau Nina Ricci est paré d'une création de la maison Lemarié. Ainsi, en 1997, l'oiseau parure est à la mode, mais il est et se doit d'être un faux oiseau. Lart du plumassier est bien de faire de faux avec du vrai; c'est à cette condition que s'effectuent le travail de l'artificialité et l'effacement de l'animal. (Fig. 13) 


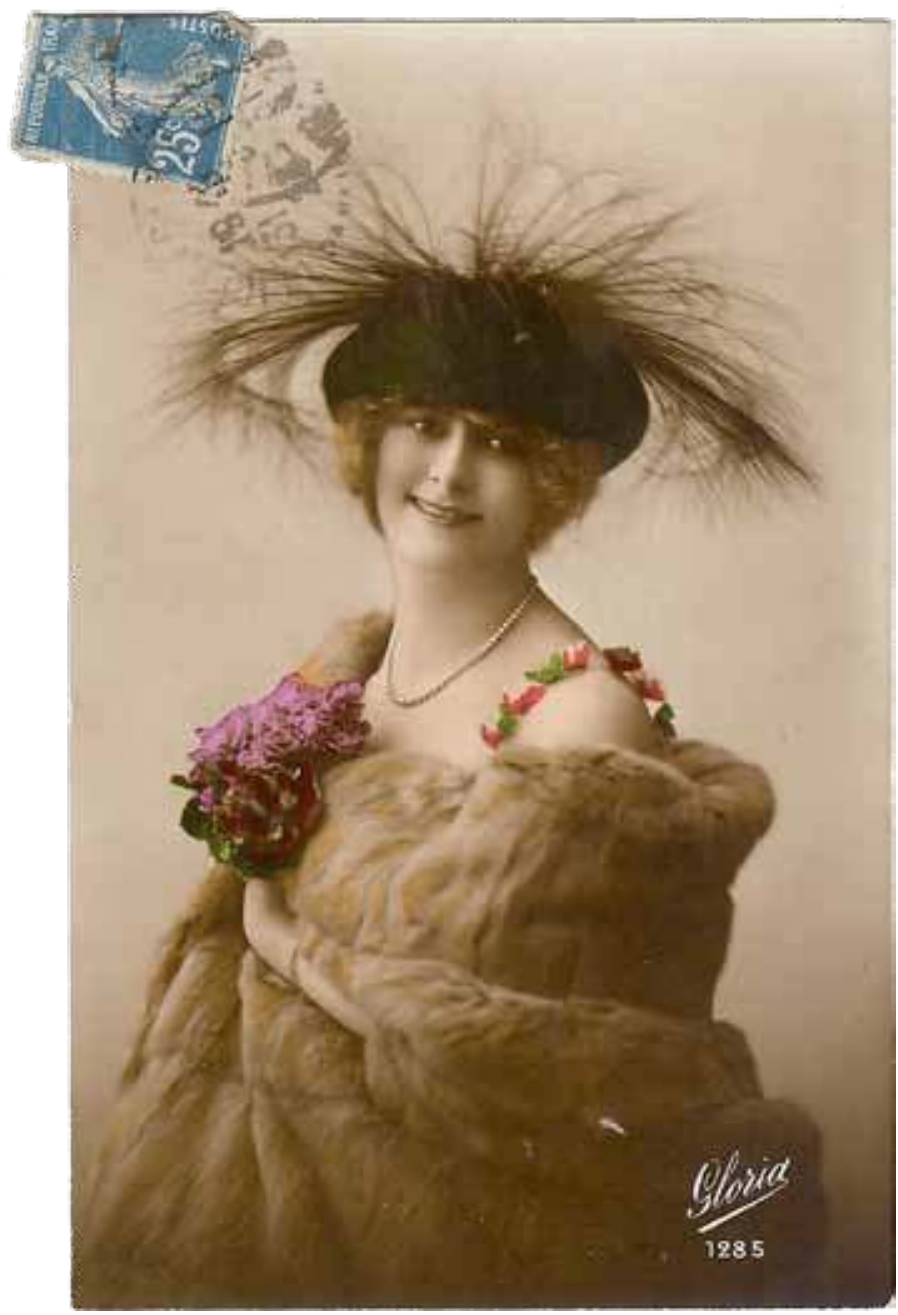

\section{Jouer avec les mots pour donner du prestige à la plume domestique}

D'autres usages renforcent ce jeu de friction entre le vrai et le faux. Les glissements lexicaux sont de ceux-là. Ils apparaissent comme une forme de ruse face à un contexte d'exploitation plumassière contraint, et par là permettent une revalorisation de la production réalisée à l'aide de plumes communes. Les plumes peuvent ainsi être rebaptisées, celle de paon traitée chimiquement acquérant le nom d' « autruche brûlée ». Cette requalification s'effectue sans obligation de transformation aucune.

Il semble exister une volonté de la part des professionnels de masquer l'origine première de la plume domestique. Dès le XIX ${ }^{e}$ siècle, les plumassiers préfèrent attribuer les noms d'espèces rares et exotiques à des espèces ordinaires pour leur conférer davantage de prestige et constituer en quelque sorte leurs lettres de noblesse. La plume de dinde ou de cigogne à sac est renommée « marabout » ou « faux marabout ». Celle de l'oie devient « cygne » (Lami 1887: 423). La plume d'émeu est rebaptisée « autruche »; celles d'autruche d'Amérique ou du nandou, autruche sauvage, comme celles du

\section{Plume, fleur et fourrure: \\ la trilogie de la maturité}

Plumes (celles d'autruches et les autres appelées les « fantaisies »), fleurs et fourrures, qui permettent de jouer sur les accessoires de mode, font bon ménage (carte postale: coll. Anne Monjaret). Cette trilogie construit à la Belle Époque les signes de l'élégance féminine, plaçant ainsi la femme du côté de la nature, mais d'une nature dressée, retravaillée. Dans les bonnes familles au XIXe siècle, si la jeune fille peut porter des fleurs, elle doit attendre le mariage pour porter des plumes et des fourrures (Knibiehler et al. 1983 : 97-98). Par ailleurs, si les productions de plumes et de fleurs sont associées, celles de plumes et de fourrures, sans se confondre, connaissent quelques similitudes dans leur traitement technique, comme leur conservation. Certaines pièces en plume sont qualifiées de fourrure. Le lexique du plumassier est riche et complexe: il y a autant de noms, sinon plus, que de volatiles répertoriés; sur un même oiseau, la région de pousse de la plume sur le corps de l'animal (tête, croupe, aigrette, collet (cou), aile, queue) et la forme de la plume elle-même (crosse (courbée), coquille (carrée), nageoire (ronde), petite, grosse, fine) appellent une terminologie spécifique. Les qualités de la plume en appellent une autre, sa transformation aussi (《 saule pleureur », « esprit », « crête », « colonel », « jet d'eau », « rafale », etc.) ainsi que sa couleur (ombrée, tachetée, marronne, amandine, blondine, écarlate, isabelle, flamboyant, souci).

(Fig. 14) coq, sont appelées « vautour », celle du héron, « aigrette » (Lami 1887: 421 ; Lacroix-Danliard 1891 : 317 ; Coffignon s.d.; Sciama 1912 : 27 ; Fourmaux 1992, $2001: 233$ ).

L'ensemble de ces désignations, pour la plupart encore d'actualité, révèle également les usages établis pour détourner les contraintes de marché (le coût puis l'interdiction de la plume exotique) tout autant que la nécessité pour ces commerçants de valoriser leurs marchandises. Ces mots, qui tiennent du registre terminologique professionnel, sont révélateurs des arguments de défense choisis par les plumassiers: ceux $\mathrm{du}$ faux-semblant, du vrai-faux pour respecter le vrai sauvage. Ces transferts de dénomination ont sans doute été renforcés avec les mises en application des conventions de sauvegarde animale qui interdisent l'usage de paradis, d'aigrette, de héron, de marabout et de vautour. Les termes sont alors conservés pour désigner d'autres espèces (Fourmaux 2001 : 233). Dès 1891, un auteur s'insurge contre ces changements de nominations qui font perdre la trace de l'origine des plumes, arguant que «malgré ces supercheries connues et ces désignations mensongères, de quelque nom qu'on les décore et de quelque pays qu'elles viennent, les plumes de parure trouvent preneurs. Vendeurs et acheteurs sont 

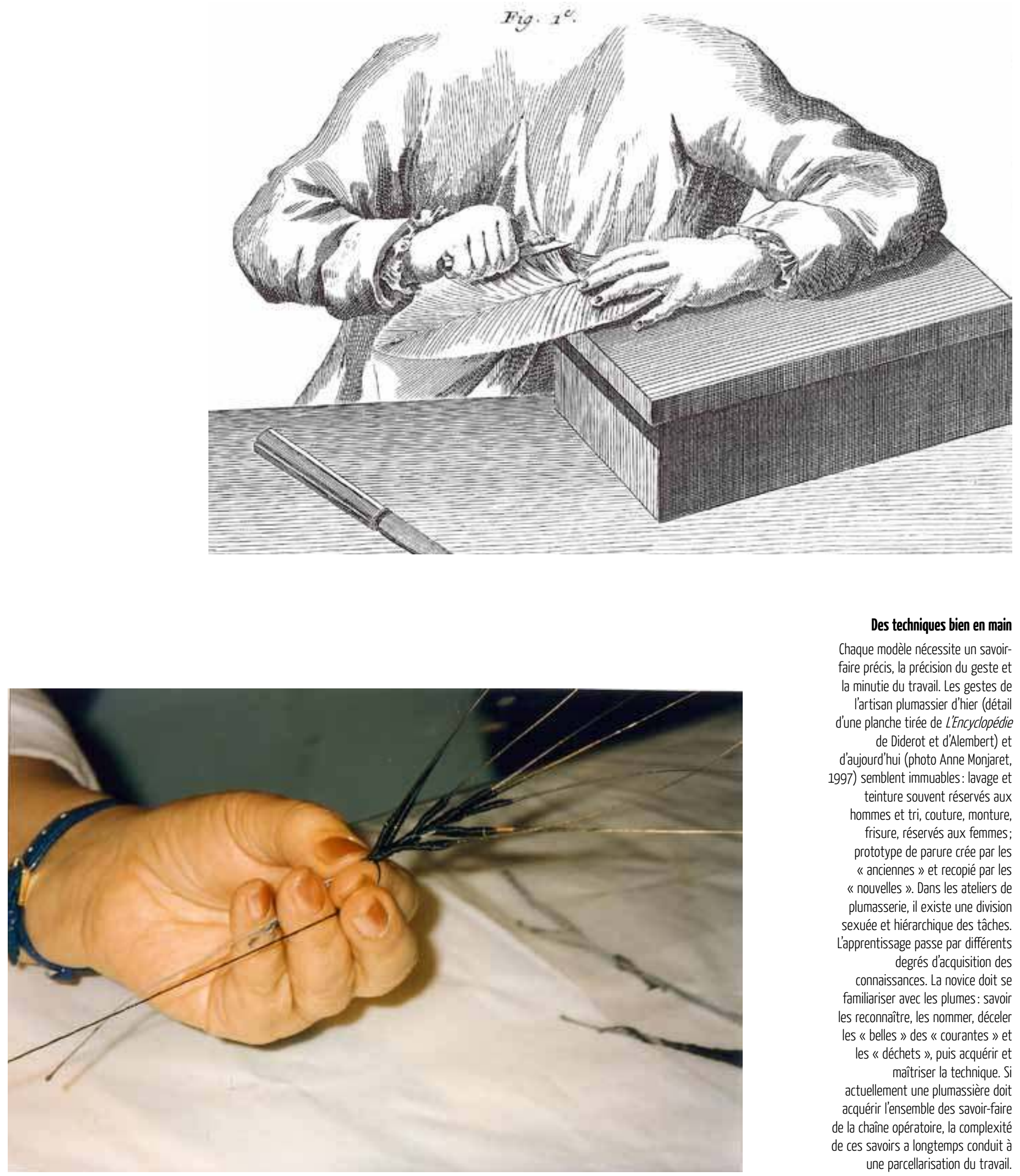

Des techniques bien en main

Chaque modèle nécessite un savoirfaire précis, la précision du geste et la minutie du travail. Les gestes de l'artisan plumassier d'hier (détail d'une planche tirée de L'Encyclopédie de Diderot et d'Alembert) et d'aujourd'hui (photo Anne Monjaret, 1997) semblent immuables: lavage et teinture souvent réservés aux hommes et tri, couture, monture, frisure, réservés aux femmes; prototype de parure crée par les « anciennes » et recopié par les « nouvelles ». Dans les ateliers de plumasserie, il existe une division sexuée et hiérarchique des tâches. L'apprentissage passe par différents degrés d'acquisition des

connaissances. La novice doit se familiariser avec les plumes: savoir les reconnaitre, les nommer, déceler les « belles » des « courantes » et

les « déchets », puis acquérir et maitriser la technique. Si actuellement une plumassière doit acquérir l'ensemble des savoir-faire de la chaîne opératoire, la complexité de ces savoirs a longtemps conduit à une parcellarisation du travail.

(Fig. 15 et 16) 


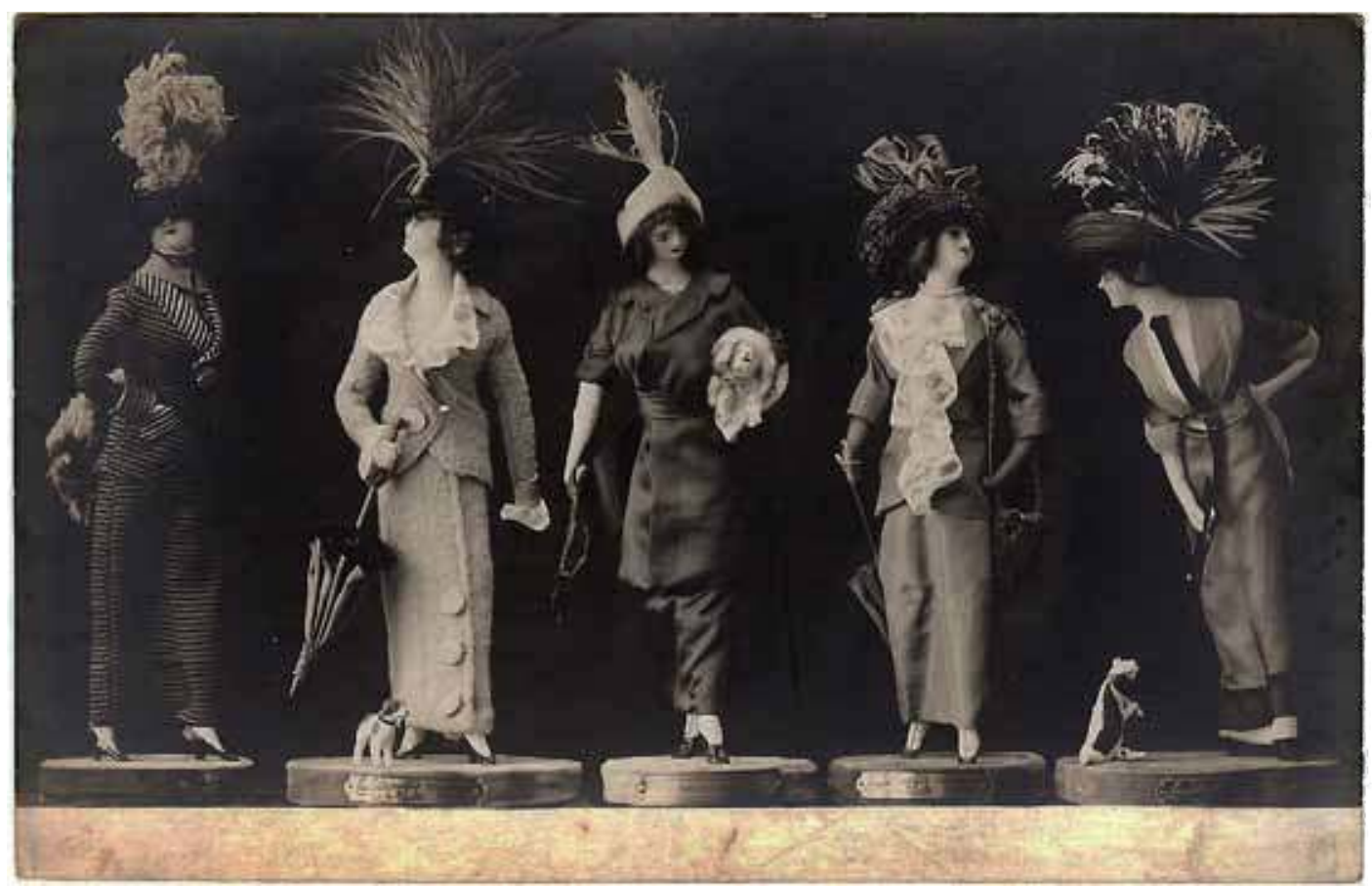

satisfaits; et il n'est guère que les pauvres bêtes qui fournissent la matière première qui n'y trouvent pas leur compte; ayant, au contraire, tout à y perdre » (Lacroix-Danliard 1891 : 316-317).

\section{$\&$}

Si le travail du plumassier s'arrêtait à la seule copie de la nature à la manière des fleuristes en fleurs artificielles, les détracteurs de ce marché manqueraient d'arguments. Ce que ces derniers reprochent aux plumassiers dès le XIX ${ }^{\mathrm{e}}$ siècle, ce sont l'emploi de plumes d'oiseaux menacés à des fins commerciales, autant que l'ostentation indécente de ces parures animalières (Fig. 17 et 18).

Exploités massivement, les oiseaux de la mode, sauvages et exotiques, apparaissent très vite comme des oiseaux de la discorde, principalement au tournant des XIX ${ }^{e}$ et XX $\mathrm{XX}^{\mathrm{e}}$ siècles. La contestation convoque alors la justification, qui devient elle-même outil de légitimation: les professionnels incriminés, de l'éleveur à l'artisan en passant par l'industriel, vont faire alliance avec des naturalistes ornithologues pour désamorcer la critique. Partisans et détracteurs d'une industrie plumassière se défendent d'être, chacun à leur manière, des protecteurs de la nature, et se répondent dans une joute argumentaire. Tous s'alarment contre les menaces qui pèsent sur certaines espèces. Pourtant, ils sont en désaccord. Leurs débats montrent la complexité des mécanismes d'une prise de conscience naturaliste, sinon écologique. 


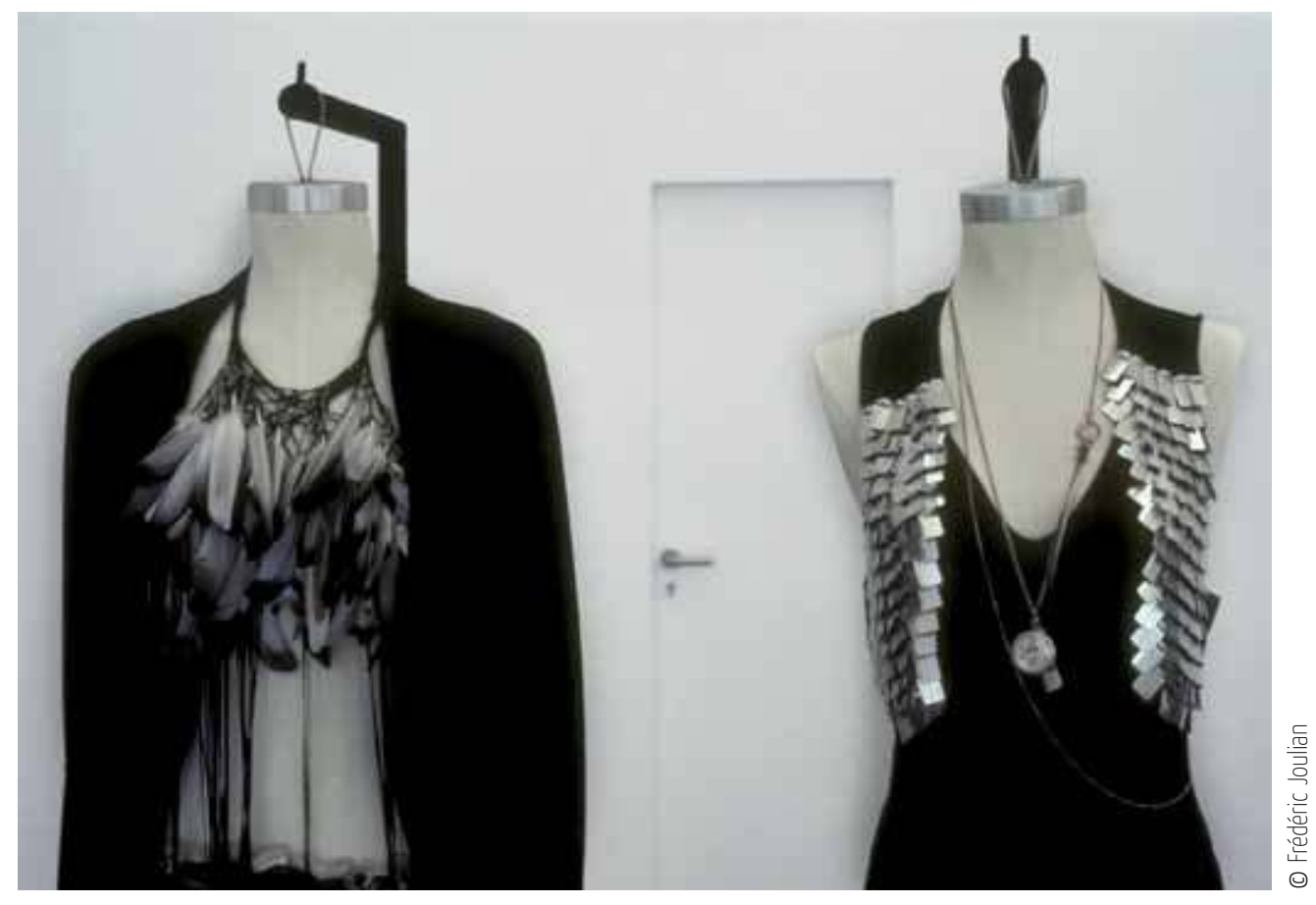

Par ailleurs, face aux conventions qui réglementent les circulations internationales de la faune, les plumassiers de la Belle Époque vont promouvoir l'élevage, adapter techniquement leurs productions en usant d'ingéniosité, transformer une plume domestique pour lui donner une apparence exotique, très prisée. Ce ne sont donc pas les conventions qui provoqueront leur déclin, bien au contraire.

Si le marché des plumes pour parure ne s'est pas complètement éteint, il est actuellement loin d'être comparable à celui de la fin du XIX et du début du $\mathrm{XX}^{\mathrm{e}}$ siècle, ayant essuyé de nombreuses crises, celles qui ont touché les maisons de mode, de couture et de spectacle. Les espèces utilisées ne sont désormais pas incluses dans la liste du CITES sauf si elles appartiennent à de vieux stocks dont les professionnels usent avec parcimonie. Mais surtout, les femmes ne portent plus que rarement des chapeaux, et encore moins agrémentés de plumes. Il faut des occasions exceptionnelles pour se parer ainsi.

Ce sont les danseuses de music-hall avec leur «truc en plume » (Fig. 19), ou les mannequins lors des défilés de haute couture, qui sont aujourd'hui les seules à arborer de telles créations, souvent ostentatoires. La plume d'ornement a été largement supplantée par la plume de spectacle, insolente et érotique. D'une certaine manière, on peut dire de la plume qu'elle est sortie de l'ordinaire féminin. Francine Fourmaux écrit qu'« elle est davantage apparentée au ridicule et reléguée dans un passé friand de surcharge » (Fourmaux 1992: 10).

Malgré ces évolutions, les techniques de fabrication sont restées identiques; le poids des traditions est lourd. Il semble que les rares plumassiers contemporains parisiens portent et défendent volontiers l'héritage laissé et revendiqué par les anciens, soucieux de préserver l'image d'une profession 


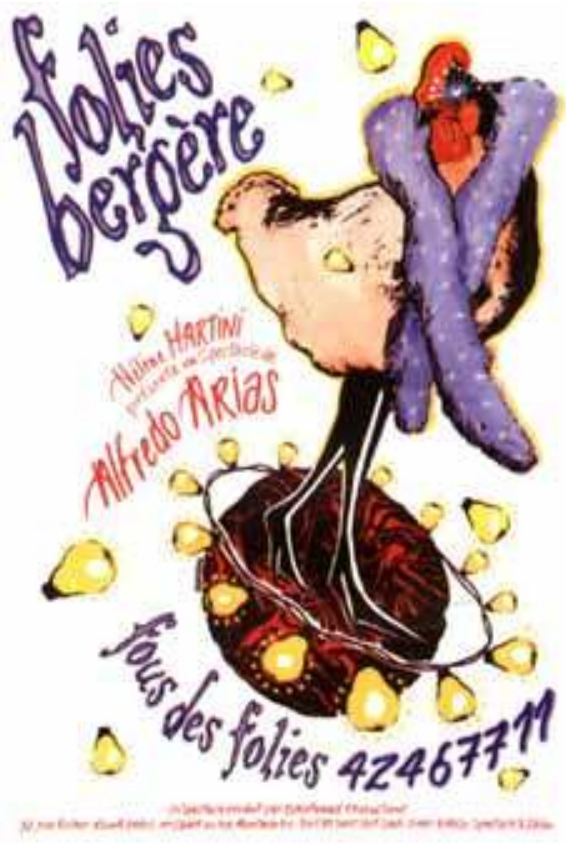

en proie à la critique dont les pratiques étaient, considéraientils, respectueuses de la nature. Pour ces héritiers, l'invention et l'innovation passent par l'acte de création, par l'originalité des motifs plus que par les moyens mobilisés pour les réaliser. Il reste qu'une page de l'histoire est tournée: les plumes de la mode ne soulèvent plus, depuis longtemps, de controverses; ces dernières sont enterrées, oubliées. Elles avaient pourtant été un moteur du changement à la Belle Époque. Ce sont aujourd'hui les fourrures portées par les élégantes qui ont pris le relais en provoquant des polémiques plus vives encore...

\section{« La Poule » au propre et au figuré}

En 1993, pour son spectacle « Fou de folie », Alfredo Arias choisit l'autodérision (Fourmaux 2005). Son affiche (conception et illustration: Bronx/Maurice Maréchal - www.bronx.fr) donne le ton: une poule avec son boa, symbole du music-hall pour représenter les filles de ces lieux de spectacle, une poule au propre et au figuré pour rappeler les registres sensuels et plus encore sexuels de ces salles des nuits parisiennes. Cette fois, la plume pare un corps nu et permet de jouer sur le caché/montré, l'érotisme féminin.

(Fig. 19)

\section{NOTES}

1. Et si l'on s'attache à toute la filière de production, il faut tenir compte également des éleveurs et des taxidermistes qui s'inscrivent dans la chaîne technique.

2. Cet article se fonde entre autres sur les résultats d'une première enquête ethnographique effectuée en 1994-1995 auprès de modistes et de merciers, et d'une seconde réalisée en 1997 auprès d'établissements parisiens fournisseurs d'articles pour la mode, plumassiers, tels que la Maison Lemairé; Maison Guillet; Ets Legeron LCT; Maison Hoguet; La société parisienne de parures et de nouveautés; Artnuptia-Maison G. Chanet; Ets Rodolphe Simon; Ets Schmid.

3. Je remercie Vanessa Manceron et Marie Roué pour leurs conseils avisés qui m’ont aidée à améliorer cet article.

4. Jules Forest explique que «pour les aigrettes, aujourd'hui, en grande partie, la cueillette de leur parure se fait surtout après la mue: on laisse vivre l'oiseau en liberté » (Forest 1898 : 780). Ces propos confortent ceux d'Edmond Lefèvre.

5. Telle la collection d'Émile Topsent, dite de «zoologie appliquée », créée en 1926 et présentée au public en 2006 à Strasbourg dans le cadre de l'exposition « Destins d'animaux - Objets et usages d'autrefois ». Cette collection constituée d'échantillons bruts et d'objets manufacturés, productions d'origine animale, était exposée dans une salle du musée zoologique.

6. Il serait intéressant de comparer dans les différents pays européens - par exemple la GrandeBretagne et l'Allemagne - concernés par les lois de protection animale, et ce à partir du milieu du XIX ${ }^{e}$ siècle, période de leurs mises en place, les modes de mobilisation des professionnels de la plume autant que ceux de leurs partisans et de leurs détracteurs. 


\section{RÉFÉRENCES}

Bollak, Léon, 1914, L'Emploi rationnel de la plume des oiseaux sauvage. Réglementation: oui; prohibition: non. Paris, Comité d'ornithologie économique.

Bolomier, Eliane, 1993, Cent ans de chapeaux 1870-1970. Catalogue, Musée du Chapeau, Chazelles-sur-Lyon.

Calmettes, Pierre, s.d. (environ 1904-1905), « Les plumes pour parures » in Excursions à travers les métiers. Paris, F. Juven: 223-229.

Celnart, Elisabeth, 1827, Manuel des dames ou l'art de la toilette suivi de l'art du modiste, du mercier et du passementier. Paris, Librairie encyclopédique de Roret.

Celnart, Elisabeth, 1838, Nouveau manuel du fleuriste artificiel ou l'art d'imiter d'après nature, suivi de l'art du plumassier. Paris, Librairie encyclopédique de Roret.

Coffignon, Ali, s.d. (fin du XIX'), Les Coulisses de la mode. Paris, Lib Illustrée, Paris Vivant.

Exposition universelle internationale de 1900 à Paris, Rapport du jury international, groupe XIII - Fils, tissus, vêtements. Deuxième partie - Classes 85 et 86 (Fleurs et plumes), Paris, Imprimerie nationale.

Forest, Jules aîné, 1898, «Industrie. La question des oiseaux. Contribution universelle des oiseaux dans l'industrie des plumes de parure », Revue scientifique, 4e série, t.19: 779-785.

Fourmaux, Francine \& Monjaret, Anne, 1993, «La fleuriste et le plumassier », Artisans de l'élégance, Catalogue, Paris, RMN, MNATP : 61-75.

Fourmaux, Francine, 1992, Etre dans la plume, Article de DEA, Université de Paris X - Nanterre.

Fourmaux, Francine, 2001, Les Filles des Folies. Ethnologie d'un music-hall parisien: usages du corps dans un espace de prodigalités, Thèse de doctorat, Université de Paris X - Nanterre.

Fourmaux Francine, 2005, «Folies-Bergère: les deux dernières revues », Ethnologie française 4: 617-626.

Franklin, Alfred, 1896, La Vie privée d'autrefois, les magasins de nouveautés. Paris, Plon, t.3.

Franklin, Alfred, 1905-1906, Dictionnaire historique des arts, métiers et professions exercés dans Paris depuis le XIII siècle, rubrique plumassiers, fleuristes, Marseille, Laffitte reprints (réimpression de l'édition de Paris-Leipzig)

Izard, Michel \& Wachtel, Nathan, 1992, «L'ethnohistoire », in Pierre Bonte \& Michel Izard, Dictionnaire de l'ethnologie et de l'anthropologie. Paris, PUF : 336-338.

Knibiehler, Yvonne, Bernos, Marcel, Ravoux-Rallo, Elisabeth \& Richard, Eliane, 1983, De la pucelle à la minette: les jeunes filles de l'âge classique à nos jours. Paris, Messidor, Temps actuels.

Lacroix-Danliard, 1891, La Plume des oiseaux: histoire naturelle et industrie, Paris, JB Baillière et fils (bibliothèque des connaissances utiles).

Lami, E.O., 1887, Dictionnaire encyclopédique et biographique de l'industrie et des arts industriels, les plumassiers. Paris, Librairie des dictionnaires.

Larousse Ménager, 1926, notice plume: 951-953.

Lefèvre, Edmond, 1914, Le Commerce et l'industrie de la plume pour parure. Paris, Ed. Edmond Lefèvre.

Monjaret, Anne, 1995, «Les métiers du chapeau au Musée nationale des arts et traditions populaires », Bulletin de liaison de la société du musée national des Arts et Traditions populaires 18: 18-21.

Monjaret, Anne, 1998, « Les modistes: de l'artisan à l'artiste, les mutations d'un corps de métier à travers le contexte de production », Ethnologie française 2: 235-246

Picard, G, 1979, Une fabrique de fleurs artificielles. Mémoire de l'École du Louvre, 2e année.

Pujol, Raymond \& Carbone, Geneviève, 1990, « L'homme et l'animal » in Jean Poirier, Histoire des mours, Paris, Gallimard (Pléiade): 1307-1388.

Schmitt, Jean-Claude, 1992, «Lanthropologie historique », in Pierre Bonte \& Michel Izard, Dictionnaire de l'ethnologie et de l'anthropologie, Paris, PUF: 339-339.

Sciama, 1912, Plumes et panaches. Un centenaire 1812-1912. Ed La Société Anonyme Sciama.

Séguret, Olivier, 1990, Entrée des fournisseurs. Paris, Ed Assouline, Maeght Ed. 


\section{RÉSUMÉ}

Plume et mode à la Belle Époque. La plume, matière première d'origine animale, a toujours été utilisée, en France et ailleurs, par les professionnels de la mode et du spectacle pour garnir et embellir les vêtements et leurs accessoires. Ce sont les plumassiers qui ont charge de transformer l'oiseau ou la plume en parure. Leurs savoirs techniques semblent traverser le temps, malgré des contextes sociaux et économiques en mutation. Les artisans d'aujourd'hui sont les héritiers des artisans d'hier. C'est le constat de cette pérennité qui a incité l'auteur, Anne Monjaret, ethnologue, à un retour en arrière, afin de mieux cerner leur héritage et de mieux saisir les conditions de sa constitution. La Belle Époque semble en cela une période charnière, une période de tension qui aura un effet sur la pratique de ce corps de métier.

En effet, au tournant du XIX et XX siècles, les oiseaux, sauvages et exotiques, ont été exploités massivement à des fins commerciales, en particulier par la mode parisienne pour garnir les chapeaux des femmes. Cet usage est très vite contesté. Détracteurs (journalistes ou hommes de lettres) et partisans s'affrontent autour de la production de l'industrie plumassière. C'est à partir de leurs écrits, et par là, des discours qu'ils ont émis à l'époque que l'auteur analyse la contestation des uns et la justification des autres. Les professionnels incriminés font alliance avec des ornithologues pour désamorcer la critique. Ils vont également promouvoir l'élevage, adapter techniquement leurs productions, transformer les plumes domestiques et leur donner une apparence exotique, fabriquer de faux oiseaux. Ils contrent ainsi en partie les conventions qui se mettent en place progressivement pour réglementer les circulations internationales d'une faune menacée d'extinction. Cet exemple montre la façon dont les intérêts humains fabriquent le destin des animaux.

Aujourd'hui encore, selon les dires des professionnels enquêtés, ce contexte passé où s'expriment respect de la nature et protection animale modèle les pratiques contemporaines des rares plumassiers qui travaillent sur la place de Paris. Si le poids des traditions reste lourd pour ces héritiers, l'invention et l'innovation passent pour eux par l'acte de création, par l'originalité des motifs plus que par les moyens mobilisés pour les réaliser. Il reste qu'une page de l'histoire est tournée; les plumes de la mode ne soulèvent plus, depuis longtemps, de controverses; ces dernières sont enterrées, oubliées. Ce sont les danseuses de music-hall avec leur « truc en plume », ou les mannequins lors des défilés de haute couture, qui sont désormais les seules à arborer de telles créations, souvent ostentatoires. Les femmes de la rue ne se parent plus de plumes. La plume d'ornement a été largement supplantée par la plume de spectacle, insolente et érotique.

\section{ABSTRACT}

Feather and Fashion in the Belle Epoque. Professionals in the fashion and show business always used feathers, raw material of animal origin, to decorate clothes and accessories in France and elsewhere. The bird or feather was transformed into a decoration by feather workers. Their technical know-how seems to have survived the passage of time, despite changing social and economic contexts. Today's artisans are heirs of yesterday's artisans. This continuity has led the ethnologist Anne Monjaret to go back in time in order to understand their heritage and the conditions that created it. The Belle Époque appears to constitute a turning point from this perspective. It was a period of tension that would affect the practices of this professional body.

In fact, at the turn of the 19th and 20th centuries, wild and exotic birds were exploited massively for commercial ends to decorate women's hats, in particular by Parisian fashion. This use was rapidly challenged. Critics (journalists or writers) and supporters (often ornithologists) confronted each other around the production of the feather workers industry. The paper analyses the challenges raised by the former and the latter's justifications in their writing and contemporary discourse. The accused professionals joined up with ornithologists to defuse criticism. They would likewise promote breeding, adapt their production technically, transform domestic feathers, give them an exotic appearance and produce false birds. Thus, to some extent they flouted conventions that were gradually instituted to regulate the international circulation of a fauna threatened with extinction. This example shows how human interests determine the destiny of animals. 
Even today, according to professional inquiries, this age-old context that respected nature and advocated animal protection still influences contemporary practices of the few Parisian feather workers. Tradition weighs heavy on these heirs, but their invention and innovation find expression in the creative act or originality of motifs rather than in the means of realization. Nonetheless, a page of history has been turned. Fashion feathers no longer invite controversies: these are buried and forgotten. Henceforth, only high fashion models or music hall dancers with their feathered "gadgets" display such ostentatious creations. Ordinary women do not decorate themselves with feathers. The feather as an ornament has long been replaced by the insolent and erotic feather for exhibition.

\section{MOTS CLÉS}

Plumassier, plume, mode, ornithologie, protection animale, réglementation

\section{KEYWORDS}

Feather worker, feather, fashion, ornithology, animal welfare, regulations 